\title{
THE STARBURST CLUSTER WESTERLUND 1: THE INITIAL MASS FUNCTION AND MASS SEGREGATION
}

\author{
Beomdu Lim ${ }^{1}$, Moo-Young Chun ${ }^{2}$, Hwankyung Sung ${ }^{1,5}$, Byeong-Gon Park ${ }^{2}$, JAe-Joon LeE ${ }^{2}$, \\ Sangmo T. Sohn ${ }^{3}$, Hyeonoh Hur ${ }^{1}$, And Michael S. Bessell ${ }^{4}$ \\ ${ }^{1}$ Department of Astronomy and Space Science, Sejong University, 98 Gunja-dong, Gwangjin-gu, Seoul 143-747, \\ Republic of Korea; bdlim1210@sju.ac.kr, sungh@sejong.ac.kr \\ ${ }^{2}$ Korea Astronomy and Space Science Institute, 776 Daedeokdae-ro, Yusung-gu, Daejeon, Republic of Korea \\ ${ }^{3}$ Space Telescope Science Institute, Baltimore, MD 21218, USA \\ ${ }^{4}$ Research School of Astronomy and Astrophysics, Australian National University, MSO, Cotter Road, Weston, ACT 2611, Australia \\ Received 2012 July 23; accepted 2012 November 19; published 2013 January 15
}

\begin{abstract}
Westerlund 1 is the most important starburst cluster in the Galaxy due to its massive star content. We have performed $B V I_{C}$ and $J K_{S}$ photometry to investigate the initial mass function (IMF). By comparing the observed color with the spectral-type-intrinsic-color relation, we obtain the mean interstellar reddening of $\langle E(B-V)\rangle=4.19 \pm 0.23$ and $\left\langle E\left(J-K_{S}\right)\right\rangle=1.70 \pm 0.21$. Due to the heavy extinction toward the cluster, the zero-age main sequence fitting method based on optical photometry proved to be inappropriate for the distance determination, while the near-infrared photometry gave a reliable distance to the cluster-3.8 kpc from the empirical relation. Using the recent theoretical stellar evolution models with rotation, the age of the cluster is estimated to be $5.0 \pm 1.0 \mathrm{Myr}$. We derived the IMF in the massive part and obtained a fairly shallow slope of $\Gamma=-0.8 \pm 0.1$. The integration of the IMF gave a total mass for the cluster in excess of $5.0 \times 10^{4} M_{\odot}$. The IMF shows a clear radial variation indicating the presence of mass segregation. We also discuss the possible star formation history of Westerlund 1 from the presence of red supergiants and relatively low luminosity yellow hypergiants.
\end{abstract}

Key words: open clusters and associations: individual (Westerlund 1) - stars: luminosity function, mass function Online-only material: color figures, machine-readable and VO tables

\section{INTRODUCTION}

Massive stars play a very significant role in the chemical evolution of a galaxy, as well as in the dynamical evolution of stellar systems. The formation mechanism of such massive stars still remains largely unknown, and should be explored in the future. The short lifetimes and rarity of massive stars make it difficult to study their evolution in detail. Currently the shape of the initial mass function (hereafter IMF) of low- to intermediate-mass stars is relatively well studied, while that of massive stars is poorly known due to their rarity. In this context, starburst clusters (or super star clusters) containing a few tens to hundreds of massive stars $\left(\geqslant 15 M_{\odot}\right)$, such as Westerlund 1 , the Arches, Quintuplet, NGC 3603, and Westerlund 2 in the Galaxy, and R136 in the Large Magellanic Cloud, are ideal laboratories to study the issues mentioned above. Furthermore, systematic studies of such clusters may provide an opportunity to understand the star formation environment in starburst galaxies. Westerlund 1 is known as the most massive open cluster in the Galaxy (Clark et al. 2005), and is one of a few highly reddened clusters observable in the optical.

Westerlund 1 (also called the Ara cluster) is a young open cluster located in the constellation of Ara. Westerlund (1961) discovered the cluster with the 26 inch Uppsala-Schmidt telescope at Mount Stromlo Observatory. From multi-wavelength photographic observations he assigned the object as a heavily reddened cluster in the Sagittarius arm containing O, B, and M type stars with an age of 3 Myr. With $2.2 \mu \mathrm{m}$ data from Price (1968), Westerlund (1968) also tested the extinction law. Since its discovery, several studies have only focused on the bright sources in the cluster using near-infrared (NIR) photometry. Borgman et al. (1970) observed 12 sources brighter than 5 mag in $K$ band and confirmed the presence of supergiants. Lockwood

\footnotetext{
5 Corresponding author:
}

(1974) also identified early- and late-type supergiants through observations with narrowband NIR filters $(0.78-1.05 \mu \mathrm{m})$ and found that $\mathrm{M}$ type supergiants were more reddened than $\mathrm{OB}$ stars. The strong infrared excess of $\mathrm{M}$ type supergiants was also found by Koornneef (1977) through photometry from the near to mid-infrared region $(2.2-20 \mu \mathrm{m})$. After a decade, Westerlund (1987) carried out photoelectric photometry and the first spectral classification of several bright stars in the cluster. Toward the end of the 20th century, Clark et al. (1998) studied two strong emission-line sources in the radio and mid-infrared.

In the 2000s, many significant discoveries were made from multi-wavelength studies. Clark \& Negueruela (2002, 2004), motivated by the results of Clark et al. (1998), found a new luminous blue variable and 11 Wolf-Rayet (WR) stars from spectroscopic studies. Subsequently, Clark et al. (2005) carried out photometric and spectroscopic observations with the $3.6 \mathrm{~m}$ New Technology Telescope, and published new spectral types for 53 members and deep photometric data down to $21 \mathrm{mag}$ in $V$. From time series observations, Bonanos (2007) published a list of variables in the direction of Westerlund 1, but only a few cluster members were included in the list. X-ray observations opened a new view of Westerlund 1. A magne$\operatorname{tar}$ (CXO J164710.2-455216), which is a probable member of Westerlund 1, was discovered by Muno et al. (2006). The existence of the magnetar implies that there have been supernova (SN) explosions more than once. Many bright X-ray sources have been found from X-ray observations (Skinner et al. 2006; Clark et al. 2008). They also discussed the stellar X-ray properties and X-ray emission mechanisms. Due to the heavy extinction and owing to the recent advances in NIR instrumentation, observations in the NIR wavelength are favored by many recent studies. Crowther et al. (2006) identified 24 (including a suspected) WR stars using NIR photometric and spectroscopic data. According to their results, the number of WR stars in Westerlund 1 reaches up to $8 \%$ of all known WR stars in the 
Galaxy. Moreover, a considerable number $(\geqslant 62 \%)$ of the stars comprise binary systems. The presence of intermediate-mass pre-main-sequence (PMS) stars was first identified with NIR photometry by Brandner et al. (2008). Unfortunately, Clark et al. (2008) could not find the NIR counterparts of several $\mathrm{X}$-ray sources, which were suspected to be low-mass PMS stars in Westerlund 1. Mengel \& Tacconi-Garman (2007) obtained the dynamical mass of $6.3 \times 10^{4} M_{\odot}$ using the integrated NIR spectra.

Interestingly, Westerlund 1 contains a substantial number of massive evolved stars at various transitional stages. The spectral types of these stars were determined in several previous studies (Clark \& Negueruela 2002, 2004; Clark et al. 2005; Crowther et al. 2006; Mengel \& Tacconi-Garman 2007; Negueruela et al. 2010; Clark et al. 2010; Dougherty et al. 2010). Thanks to the richness of massive transitional objects, it is possible to study the evolution of massive stars as well as to observationally test their mass-loss rate before undergoing an SN explosion. Most stars detected in the radio continuum observations do not show a significant mass loss that could explain the current mass of WR stars, while a bright radio source, W9, shows a higher mass-loss rate and an extended nebula that is indicative of heavy mass loss in the past (Dougherty et al. 2010). It is suggested that short-lived and episodic mass-loss events due to unknown mechanisms are required to explain the current mass of these evolved stars. Several previous studies have attempted to monitor several evolved stars spectroscopically to understand the properties of variability as well as to constrain the initial mass of the magnetar (Ritchie et al. 2009a, 2009b, 2010; Clark et al. 2010, 2011). Recently, Koumpia \& Bonanos (2012) determined the fundamental parameters of four eclipsing binaries in Westerlund 1.

Despite many detailed studies, the distance to Westerlund 1 is still being debated. Early studies, e.g., Westerlund (1961), Borgman et al. (1970), and Piatti et al. (1998), favored a closer distance (1.1-1.4 kpc) from the brightness of supergiants. Brandner et al. (2008) determined a distance of $3.6 \pm 0.2 \mathrm{kpc}$ from NIR photometry and theoretical isochrones. A refined analysis by Gennaro et al. (2011) suggested a distance of $4.0 \pm 0.2 \mathrm{kpc}$. Kothes \& Dougherty (2007) independently derived a distance of $3.9 \pm 0.7 \mathrm{kpc}$ based on their new Galactic rotation curve from $\mathrm{H}$ I observation. Koumpia \& Bonanos (2012) obtained a distance of $3.7 \pm 0.6 \mathrm{kpc}$ using the binary method. Clark et al. (2005), Crowther et al. (2006), and Negueruela et al. (2010) prefer a distance of $5 \mathrm{kpc}$, which is well supported by their observational data. Although the wide range in the distance is now converging to $4.0-5.0 \mathrm{kpc}$, the exact distance to Westerlund 1 remains the most crucial parameter in the study of Westerlund 1.

The IMF is the most important tool with which to understand star formation processes, the formation and evolution of stellar systems, the contribution of stellar mass to the host galaxy, the overall stellar properties of unresolved remote galaxies, and the evolution of galaxies. In particular, the IMF of starburst clusters could give clues to understanding the active star-forming conditions in starburst galaxies, and therefore it is possible to extend this view to the formation stage of the Milky Way. Unfortunately, such clusters in the Galaxy are mostly distant and highly reddened. Detailed studies are very difficult because of these observational limitations. In spite of such difficulties, several studies on the IMF of the starburst clusters or well-known active star-forming regions have been made. The slope of the $\operatorname{IMF}(\Gamma)$ is -0.7 to -1.1 for the Arches cluster (Figer et al.
1999; Kim et al. 2007; Espinoza et al. 2009), -1.3 to -1.4 for the R136 cluster (Massey \& Hunter 1998), -0.7 to -0.9 for NGC 3603 (Eisenhauer et al. 1998; Sung \& Bessell 2004; Stolte et al. 2006), -1.3 for Trumpler 14 and 16 in the $\eta$ Carina nebula (Hur et al. 2012), -1.3 for NGC 6530 (Sung et al. 2000), and -1.2 for NGC 6231 (Sung et al. 1998). The slope of the IMF of Westerlund 1 is known to be -1.3 to -1.4 (Brandner et al. 2008; Gennaro et al. 2011). Although they statistically derived the IMF of Westerlund 1 from deep NIR data, their data are unsuitable for the study of the IMF of massive stars due to saturation of the bright stars. Moreover, the masses inferred from NIR photometry are rather uncertain, especially for the massive stars, due to the lower resolution of NIR colors. Fortunately, the starburst cluster Westerlund 1 is still accessible for observations in the optical passband with a $4 \mathrm{~m}$ class telescope, and therefore it is possible to study the shape of the IMF of the massive part in detail.

Open clusters, being stellar systems, are subject to dynamical evolution. To understand dynamical evolution in open clusters, many models and numerical simulations have focused on mass segregation. As mass segregation is predicted as a consequence of energy equipartition, one could expect that open clusters younger than the relaxation time are unlikely to show mass segregation. However, mass segregation has been found even in young open clusters, such as the Orion Nebula Cluster (Hillenbrand \& Hartmann 1998) and NGC 6231 (Raboud \& Mermilliod 1998; Sung et al. 2013). Several recent attempts have been made to understand such mass segregation in the context of dynamical evolution (McMillan et al. 2007; Moeckel \& Bonnell 2009; Allison et al. 2009, 2010). They showed that substructure and subvirial conditions at early stages of cluster formation can lead to mass segregation as a consequence of dynamical evolution within a very short timescale. On the other hand, primordial mass segregation has also been suggested by competitive accretion (Bonnell \& Davies 1998; Bonnell et al. 1998; Bonnell \& Bate 2006) or core coalescence (Dib et al. 2008). In this context, the dynamical evolution of young starburst clusters is a very interesting topic because of their large numbers of massive stars. A feature indicative of mass segregation below $32 M_{\odot}$ has been found by Brandner et al. (2008), who showed a difference in the half-mass radii of high- and lowmass stars. Gennaro et al. (2011) supplied strong evidence of mass segregation from the spatial variation of the IMF. However, any mass segregation among the most massive stars, which can cause a violent relaxation, was not studied, because their data were confined to stars with masses below $40 M_{\odot}$.

The purpose of this study of Westerlund 1 is to determine a reliable distance, reddening, and age of the cluster, as well as to derive its IMF. In addition, we will investigate the mass segregation of the most massive stars, which dynamically play a great role in the cluster. To achieve our aims, optical and NIR photometric data were obtained using $4 \mathrm{~m}$ class telescopes. In Section 2, we describe our observations and data sets. In Section 3, we present several photometric diagrams and determine the fundamental parameters of Westerlund 1, such as the radius, reddening, distance, and age. The IMF and mass segregation are addressed in Sections 4 and 5, respectively. We present discussions on several outstanding questions in Section 6. Finally, the summary is in Section 7.

\section{OBSERVATIONS}

\subsection{Optical Data}

The observations of Westerlund 1 were carried out on 2009 March 28 at the Cerro Tololo Inter-American Observatory 
Table 1

Atmospheric Extinction Coefficients

\begin{tabular}{lccc}
\hline \hline Filter & $k_{1}(2009$ Mar 28) & $k_{1}(2009$ Mar 29) & $k_{2}$ \\
\hline$B$ & $0.244 \pm 0.010$ & $\ldots$ & $0.026 \pm 0.001$ \\
$V$ & $0.141 \pm 0.006$ & $0.134 \pm 0.014$ & $\ldots$ \\
$I_{C}$ & $0.053 \pm 0.011$ & $0.075 \pm 0.018$ & $\ldots$ \\
\hline
\end{tabular}

(CTIO) in Chile. All the images were taken with the Mosaic II $\operatorname{CCD}\left(36^{\prime} \times 36^{\prime}\right)$ attached to the Blanco $4 \mathrm{~m}$ telescope. The seeing was about 0 '. 8 . Harris $B V I_{C}$ and Sloan Digital Sky Survey (SDSS) $u$ filters were used. The angular size of the cluster is so compact that we could place the cluster on the best chip (chip 6) among eight chips, where the field of view of an individual chip is about $9^{\prime} \times 18^{\prime}$. Given its severe extinction, special care was paid to the exposure times $10 \mathrm{~s}$ and $150 \mathrm{~s} \times 3$ in $I, 300 \mathrm{~s} \times 3$ in $V, 600 \mathrm{~s} \times 3$ in $B$, and $200 \mathrm{~s}, 600 \mathrm{~s}$, and $1200 \mathrm{~s}$ in $u$, respectively. In order to transform instrumental magnitudes to standard magnitude reliably, many standard stars in the Landolt standard fields (Landolt 1992) were observed at a wide range of air masses. We made use of IRAF $^{6}$ packages, MSCRED and DAOPHOT, to eliminate the instrumental signatures and to carry out point spread function (PSF) photometry.

We performed aperture photometry for the standard stars with an aperture size of $10^{\prime \prime}$. The instrumental magnitudes were transformed to the standard system using the following equation:

$$
\begin{aligned}
M_{\lambda}= & m_{\lambda}+\eta_{\lambda} \cdot C_{0}-\left(k_{1, \lambda}-k_{2, \lambda} \cdot C_{0}\right) \cdot X+\alpha_{\lambda} \cdot \hat{U T} \\
& +\beta_{\lambda} \cdot \hat{x}_{\mathrm{CCD}}+\gamma_{\lambda} \cdot \hat{y}_{\mathrm{CCD}}+\zeta_{\lambda},
\end{aligned}
$$

where $M_{\lambda}, m_{\lambda}, \eta_{\lambda}, C_{0}, k_{1, \lambda}, k_{2, \lambda}, \alpha_{\lambda}$, and $\zeta_{\lambda}$ represent the standard magnitude, the instrumental magnitude, the transformation coefficient, the standard color index, the primary extinction coefficient, the secondary extinction coefficient, the coefficient for the time variation of photometric zero point, and the photometric zero point for a given filter, respectively. $\beta_{\lambda}$ and $\gamma_{\lambda}$ denote the coefficients of spatial variation in magnitude per 1000 pixels, which will be addressed below. We adopted the Stetson compilations of the Landolt standard star fields (Stetson 2000) as the standard system. Using the weighted least-squares method, the atmospheric extinction coefficient, the transformation coefficient, and the coefficients for spatial variation were determined. We present the atmospheric extinction coefficients of the $B V I_{C}$ filters for two nights in Table 1. The transformation relations of each band and chip were determined in the form of one or a combination of two straight lines as shown in Figure 1. In the $V$ transformation, the spectral features due to $\mathrm{TiO}$ band in late-type stars make the transformation relation, with respective to $V-I$, flatter, as shown in Figure 1 (see also Sung \& Bessell 2000; Lim et al. 2009). In the case of Westerlund 1, the single relation (dashed line in the figure) extrapolated to extreme red color is adopted because the slope in the spectral energy distribution for OB stars gradually declines against the reddening effect with wavelength. On the other hand, we faced a severe problem with the so-called red leak in the $U$ transformation. Highly reddened stars, which may be probable members of Westerlund 1, were too bright and showed double-peak profiles in the $u$ images due to the red leak of the SDSS $u$ filter. It is almost impossible to correct for this amount of red leak due to a lack of standard

\footnotetext{
6 Image Reduction and Analysis Facility is developed and distributed by the National Optical Astronomy Observatory, which is operated by the Association of Universities for Research in Astronomy, Inc., under operative agreement with the National Science Foundation.
}

stars with extreme red color. More details are addressed in Appendix A.

Another remarkable feature is a systematic variation in magnitude along each axis of a given CCD chip. A similar feature for the CFH12K has been reported in Sung et al. (2008; see also Magnier \& Cuillandre 2004). Figure 2 represents the spatial variations with respect to the $x_{\mathrm{CCD}}$ or $y_{\mathrm{CCD}}\left(\hat{x}_{\mathrm{CCD}} \equiv\right.$ $\left.x_{\mathrm{CCD}} / 1000, \hat{y}_{\mathrm{CCD}} \equiv y_{\mathrm{CCD}} / 1000\right)$. The pattern of the spatial variation along the $x_{\mathrm{CCD}}$ axis seems to be random, and the maximum variation is about 0.03 mag for chip 8 . On the other hand, the variation along the $y_{\mathrm{CCD}}$ axis is very conspicuous and systematic. The slope $\gamma$ for chips $1-4$ is very large (up to $0.07 \mathrm{mag}$ ), and is larger than that for chips 5-8. This may be related to the spatial variation of the focal plane of the Blanco $4 \mathrm{~m}$ telescope. After correcting for the spatial variations, reliable transformation coefficients for each chip could be determined. We summarize the transformation coefficients, the coefficients for spatial variation, and photometric zero points for all chips in Table 2.

Recently three optical photometric studies, Piatti et al. (1998), Clark et al. (2005), and Bonanos (2007), were carried out in the Landolt standard system $\left(U B V R_{C} I_{C}\right)$. We compared our data with these previous studies and present the results in Table 3. Although the stars used in the comparison are mostly evolved stars and therefore may be suspected variables, the photometric data of Piatti et al. (1998) and Clark et al. (2005) show such large differences in both $V$ magnitude and color indices, which we attribute the discrepancies to their standard transformation. This is particularly so for Piatti et al. (1998) because errors in the extinction coefficients are very large relative to the values themselves. We suspect that they observed Westerlund 1 in nonphotometric conditions or they observed only a small number of standard stars. Clark et al. (2005), on the other hand, gave no details concerning their standard transformations but since their observation was carried out in queue mode, it seems likely that an insufficient number of red standard stars were used to determine the extinction and transformation coefficients. The $V$ magnitudes of Bonanos (2007) are in good agreement with ours; however, it is difficult to make a reliable conclusion on the consistency in the colors based on only two or three stars in common.

The photometric data for eight bright stars saturated in our data are taken from Piatti et al. (1998; W32 and W243) and Clark et al. (2005; W4, W7, W8a, W16a, W33, and W57a) after correcting for the differences in photometric zero points. We present the finder chart for the stars brighter than $V=20.5 \mathrm{mag}$ in Figure 3 and photometric data in Table 4. The filled symbols and star symbols in the figure represent stars with spectral type and luminosity class in Negueruela et al. (2010), Clark et al. (2010), Mengel \& Tacconi-Garman (2007), and Crowther et al. (2006). The evolved stars in transitional stages are substantially concentrated in a small area.

\subsection{Near-infrared Data}

The NIR data were obtained using the IRIS2 detector on the Australian Astronomical Telescope (AAT) on 2011 June 25. IRIS2 used a Rockwell Science Hawaii-1 HgCdTe 1K infrared detector, covering 7!.7 $\times$ 7!.7 field of view with a 0.45 pixel scale. Two sets of exposure times were used-long: $10 \mathrm{~s} \times 10$ readout $\times 9$ dithering in $J, 7 \mathrm{~s} \times 10$ readout $\times 9$ dithering in $K_{S}$; and short: $1 \mathrm{~s} \times 10$ readout $\times 9$ dithering in both $J$ and $K_{S}$. The basic data reduction was primarily done using ORAC-DR in the Starlink package. We subtracted dark and bias from each 


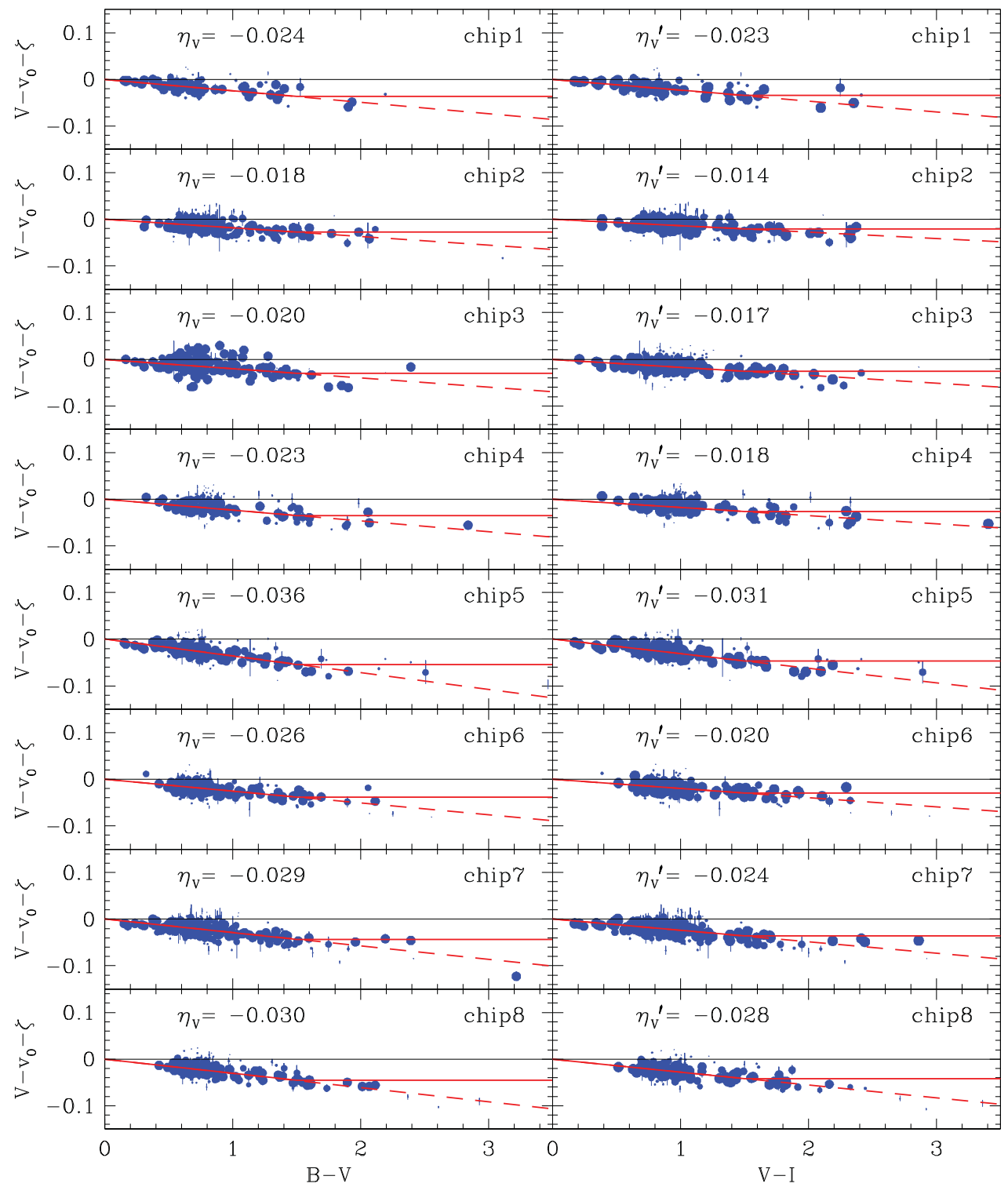

Figure 1. Transformation relations of the Mosaic II CCD in the $V$ band with respect to $B-V$ and $V-I$. The size of the dots depends on the reliability of a given standard star. All the slopes are negative with a break at $1.5 \mathrm{mag}$ in both color indices. The details of the transformation relations are addressed in the text. We adopt the single slope (dashed line) extrapolated to red colors because our main targets are highly reddened OB stars.

(A color version of this figure is available in the online journal.)

individual dithered image and then normalized them using a flat image. The flat image was generated by median combining the dithered images. Then the images were cross-correlated and combined to produce the final image.

We carried out PSF photometry using DAOPHOT. The instrumental magnitudes and colors were transformed to the Two Micron All Sky Survey (2MASS) photometric system (Skrutskie et al. 2006) using 78 stars in $J$ and 187 stars in $K_{S}$. As the photometry of bright stars are affected by the nonlinearity of the IRIS2 detector, and the photometric errors of faint stars in the 2MASS catalog are large, the differences relative to the 2MASS photometry were calculated only for the stars with $11 \mathrm{mag} \leqslant K_{S} \leqslant 13 \mathrm{mag}$. The differences were $\left\langle\Delta K_{S}\right\rangle=0.030 \pm 0.097$ and $\left\langle\Delta\left(J-K_{S}\right)\right\rangle=0.000 \pm 0.122$. Given the errors of the NIR photometry, our photometric data are well consistent with the 2MASS system.

\section{PHOTOMETRIC DIAGRAMS}

The highly reddened cluster Westerlund 1 reveals unusual color-magnitude diagrams (CMDs) in Figure 4 due to the presence of many evolved stars, such as OB supergiants (OBSGs), yellow hypergiants (YHGs), red supergiants (RSGs), luminous blue variables (LBVs), and WR stars. In the CMDs, large dots, open triangles, open squares, open pentagons, and stars represent OBSGs, YHGs, RSGs, LBVs, and WRs, respectively. It would be very useful to compare the stellar content in these CMDs with that of other clusters with similar ages. We therefore superimpose the reddened zero-age main sequence (ZAMS) relation (Sung \& Bessell 1999) and the members of NGC 6231 (Sung et al. 1998) by adopting the mean reddening and distance modulus of Westerlund 1 determined in Section 3.3. The positions of OBSGs of NGC 6231 are well matched to those of 


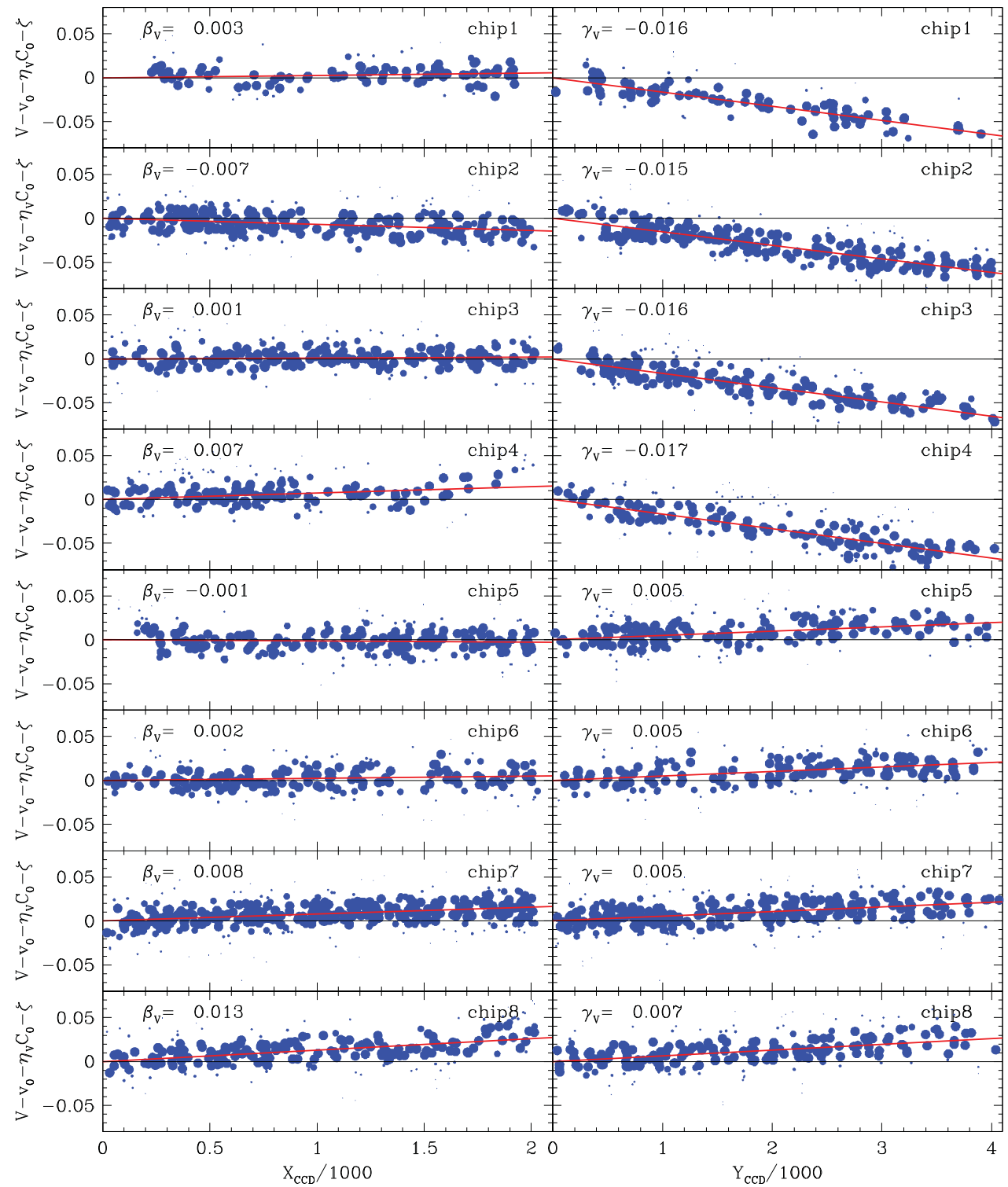

Figure 2. Spatial variation of the $V$ magnitude with respect to the $X_{\mathrm{CCD}}$ (left) or $Y_{\mathrm{CCD}}$ (right) coordinates of the Mosaic II CCD. The coordinates of the standard stars on each CCD chip have been divided by 1000 pixel. The variation along $X_{\mathrm{CCD}}$ is less significant than that along $Y_{\mathrm{CCD}}$. Among eight chips, chip 8 shows the largest variation of up to $0.03 \mathrm{mag}$ along the $X$-axis. The variations for the $Y$-axis are much more prominent in the range between -0.07 mag and 0.02 mag. The sign of the slopes is random for the $X$-axis, while it is systematic for the $Y$-axis, i.e., negative for chips $1-4$ and positive for chips 5-8.

(A color version of this figure is available in the online journal.)

Westerlund 1. The probable main sequence (MS) members of Westerlund 1 are barely seen at $V \geqslant 21 \mathrm{mag}$ in the $(V, V-I)$ diagram.

We present the NIR $\left(K_{S}, J-K_{S}\right)$ CMDs in Figure 5. To help distinguish the different sequences of Westerlund 1 stars, we plot in the middle panel of the figure, only the stars within a radius of $r=2.5$ from the center. The foreground stars in the Sagittarius-Carina arm constitute the bluest sequence. As shown in Figure 4 of Gennaro et al. (2011), the turn-on point from the PMS to the MS is found near $K_{S} \sim 15 \mathrm{mag}$, and the sequence of MS and evolved stars is stretched out within a narrow color range $\left(J-K_{S}=1-2 \mathrm{mag}\right)$. An open circle is drawn around the X-ray sources detected by Clark et al. (2008) in Figures 4 and 5. Most of the evolved stars, in particular OBSGs and WRs, are X-ray emission objects. Since PMS stars are also known as X-ray sources, we attempted to trace the PMS locus in Figure 5 by identifying the NIR counterpart of X-ray emission objects. However, only a few X-ray emission stars have NIR counterparts in the expected PMS locus. We agree with Clark et al. (2008) that many of X-ray-emitting PMS stars are fainter than $K_{S} \sim 16$ mag.

In this section, we also present the results from completeness tests and determine the fundamental parameters, such as radius, reddening, distance, and age.

\subsection{Radius of Westerlund 1}

The surface density profile of a cluster is one of the tools used to determine the radius of a cluster as well as to diagnose the dynamical structure of the cluster. The profiles also allow us to 
Table 2

Coefficients for Mosaic II Data

\begin{tabular}{|c|c|c|c|c|c|c|}
\hline Filter & Chip & $\eta_{1}$ & $\eta_{2}{ }^{\mathrm{a}}$ & $\beta$ & $\gamma$ & $\zeta(2009$ Mar 28) \\
\hline \multirow{8}{*}{$B$} & 1 & $0.046 \pm 0.008$ & $\cdots$ & $-.012 \pm 0.003$ & $-.018 \pm 0.002$ & $25.481 \pm 0.009$ \\
\hline & 2 & $0.041 \pm 0.006$ & $\ldots$ & $-.005 \pm 0.003$ & $-.013 \pm 0.001$ & $25.444 \pm 0.010$ \\
\hline & 3 & $0.034 \pm 0.005$ & $\cdots$ & $0.004 \pm 0.002$ & $-.011 \pm 0.001$ & $25.432 \pm 0.007$ \\
\hline & 4 & $0.116 \pm 0.003$ & $\ldots$ & $0.003 \pm 0.003$ & $-.013 \pm 0.002$ & $25.404 \pm 0.009$ \\
\hline & 5 & $0.058 \pm 0.005$ & $\ldots$ & $-.010 \pm 0.003$ & $0.001 \pm 0.002$ & $25.412 \pm 0.010$ \\
\hline & 6 & $0.063 \pm 0.004$ & $\ldots$ & $-.006 \pm 0.003$ & $0.003 \pm 0.001$ & $25.383 \pm 0.009$ \\
\hline & 7 & $0.057 \pm 0.003$ & $\ldots$ & $0.010 \pm 0.002$ & $0.007 \pm 0.001$ & $25.361 \pm 0.009$ \\
\hline & 8 & $0.087 \pm 0.003$ & $\cdots$ & $0.007 \pm 0.003$ & $-.001 \pm 0.002$ & $25.376 \pm 0.010$ \\
\hline \multirow{8}{*}{$\begin{array}{l}V \\
(B-V)\end{array}$} & 1 & $-.025 \pm 0.002$ & 0.000 & $0.003 \pm 0.001$ & $-.016 \pm 0.001$ & $25.695 \pm 0.009$ \\
\hline & 2 & $-.018 \pm 0.002$ & 0.000 & $-.007 \pm 0.001$ & $-.015 \pm 0.001$ & $25.692 \pm 0.009$ \\
\hline & 3 & $-.020 \pm 0.002$ & 0.000 & $0.001 \pm 0.001$ & $-.016 \pm 0.001$ & $25.676 \pm 0.007$ \\
\hline & 4 & $-.023 \pm 0.002$ & 0.000 & $0.007 \pm 0.001$ & $-.017 \pm 0.001$ & $25.688 \pm 0.009$ \\
\hline & 5 & $-.036 \pm 0.002$ & 0.000 & $-.001 \pm 0.001$ & $0.005 \pm 0.001$ & $25.647 \pm 0.010$ \\
\hline & 6 & $-.026 \pm 0.003$ & 0.000 & $0.003 \pm 0.001$ & $0.005 \pm 0.001$ & $25.627 \pm 0.010$ \\
\hline & 7 & $-.029 \pm 0.001$ & 0.000 & $0.008 \pm 0.001$ & $0.005 \pm 0.001$ & $25.622 \pm 0.009$ \\
\hline & 8 & $-.030 \pm 0.002$ & 0.000 & $0.013 \pm 0.001$ & $0.007 \pm 0.001$ & $25.627 \pm 0.009$ \\
\hline \multirow{8}{*}{$\begin{array}{l}V \\
(V-I)\end{array}$} & 1 & $-.023 \pm 0.003$ & 0.000 & $0.003 \pm 0.001$ & $-.016 \pm 0.001$ & $25.697 \pm 0.008$ \\
\hline & 2 & $-.014 \pm 0.003$ & 0.000 & $-.007 \pm 0.001$ & $-.015 \pm 0.001$ & $25.690 \pm 0.008$ \\
\hline & 3 & $-.017 \pm 0.003$ & 0.000 & $0.001 \pm 0.001$ & $-.016 \pm 0.001$ & $25.676 \pm 0.008$ \\
\hline & 4 & $-.018 \pm 0.004$ & 0.000 & $0.007 \pm 0.001$ & $-.017 \pm 0.001$ & $25.685 \pm 0.009$ \\
\hline & 5 & $-.031 \pm 0.003$ & 0.000 & $-.001 \pm 0.001$ & $0.005 \pm 0.001$ & $25.646 \pm 0.010$ \\
\hline & 6 & $-.020 \pm 0.003$ & 0.000 & $0.003 \pm 0.001$ & $0.005 \pm 0.001$ & $25.625 \pm 0.010$ \\
\hline & 7 & $-.024 \pm 0.002$ & 0.000 & $0.008 \pm 0.001$ & $0.005 \pm 0.001$ & $25.622 \pm 0.009$ \\
\hline & 8 & $-.028 \pm 0.003$ & 0.000 & $0.013 \pm 0.001$ & $0.007 \pm 0.001$ & $25.630 \pm 0.009$ \\
\hline \multirow{8}{*}{$I_{C}$} & 1 & $-.016 \pm 0.002$ & $\cdots$ & $-.003 \pm 0.002$ & $-.018 \pm 0.001$ & $25.131 \pm 0.010$ \\
\hline & 2 & $-.025 \pm 0.001$ & $\ldots$ & $-.013 \pm 0.001$ & $-.016 \pm 0.001$ & $25.109 \pm 0.009$ \\
\hline & 3 & $-.012 \pm 0.003$ & $\ldots$ & $0.001 \pm 0.001$ & $-.022 \pm 0.001$ & $25.111 \pm 0.010$ \\
\hline & 4 & $-.014 \pm 0.002$ & $\cdots$ & $0.001 \pm 0.001$ & $-.015 \pm 0.001$ & $25.100 \pm 0.010$ \\
\hline & 5 & $-.020 \pm 0.002$ & $\cdots$ & $-.008 \pm 0.001$ & $0.006 \pm 0.001$ & $25.081 \pm 0.010$ \\
\hline & 6 & $-.021 \pm 0.002$ & $\cdots$ & $-.010 \pm 0.001$ & $0.004 \pm 0.001$ & $25.047 \pm 0.010$ \\
\hline & 7 & $-.010 \pm 0.001$ & $\ldots$ & $0.014 \pm 0.001$ & $0.006 \pm 0.001$ & $25.011 \pm 0.010$ \\
\hline & 8 & $-.019 \pm 0.002$ & $\ldots$ & $0.008 \pm 0.001$ & $0.003 \pm 0.001$ & $25.050 \pm 0.010$ \\
\hline
\end{tabular}

Note. ${ }^{\mathrm{a}} \eta_{2}$ for $V$ represents the transformation coefficient for $B-V>1.5$ and $V-I>1.5$.

Table 3

Comparison of Photometry

\begin{tabular}{|c|c|c|c|c|c|c|}
\hline Paper & $\Delta V^{\mathrm{a}}$ & $N(m)^{\mathrm{b}}$ & $\Delta(B-V)^{\mathrm{a}}$ & $N(m)^{\mathrm{b}}$ & $\Delta(V-I)^{\mathrm{a}}$ & $\overline{N(m)^{\mathrm{b}}}$ \\
\hline Piatti et al. (1998) & $-1.06 \pm 0.13$ & $22(2)$ & & & $0.24 \pm 0.13$ & $11(0)$ \\
\hline Clark et al. (2005) & $-0.42 \pm 0.10$ & $48(3)$ & $0.12 \pm 0.15$ & $45(2)$ & $0.21 \pm 0.08$ & $33(2)$ \\
\hline Bonanos (2007) & $-0.05 \pm 0.06$ & $19(1)$ & $-0.02 \pm 0.01$ & $2(0)$ & $0.61 \pm 0.10$ & $3(0)$ \\
\hline
\end{tabular}

Notes.

${ }^{\text {a }}$ Others - This.

${ }^{\mathrm{b}} N$ and $m$ represents number of compared stars and excluded stars.

simulate realistically a model cluster regardless of dynamical models. In this section, we determine the radius of Westerlund 1 using the surface density profiles.

To simplify, we assume a circular shape of Westerlund 1 and assign the densest position as the center of the cluster. The width of a ring is set to 100 pixels (equivalent to 0.45 ), and the inner radius of each annuli are increased by 100 pixels. To check the difference in surface density profiles with brightness, the surface density profiles in the $I$ band are calculated for a bin size of 1 mag as in Sung et al. (1999). The error in the surface density is assumed to follow Poisson statistics, i.e., $\epsilon= \pm \sqrt{N} / S$ (where $N$ and $S$ are the number of stars and the area of the annulus, respectively). We present the surface density profiles in Figure 6 where an appropriate constant is added to each profile to show the difference in profiles clearly.
The bright stars are obviously concentrated in the center of the cluster. The surface density of bright stars is definitely higher in the inner region, while less significant in the outer region. The contrast of surface density between the inner and outer regions deceases as the brightness of stars decreases, and finally the profiles of faint stars are dominated by Poisson noise. We attribute the lack of faint stars in the inner region either to the incompleteness of photometry or to dynamical mass segregation, or both. The distance from the center to the point where the surface density converges to a constant level is about 2.5 regardless of brightness. In order to check the biases from the environmental effect in our analysis, we have conducted the same procedure for X-ray sources published in Clark et al. (2008) as well as our NIR data. Only the X-ray emission stars with $V-I>4.0$ mag were used to avoid non-uniformity of 
Table 4

Photometric Data

\begin{tabular}{|c|c|c|c|c|c|c|c|c|c|c|c|c|c|c|c|c|}
\hline $\mathrm{ID}^{\mathrm{a}}$ & $\alpha_{\mathrm{J} 2000}$ & $\delta_{\mathrm{J} 2000}$ & $V$ & $I$ & $V-I$ & $B-V$ & $U-B$ & $\epsilon_{V}$ & $\epsilon_{I}$ & $\epsilon_{V-I}$ & $\epsilon_{B-V}$ & $\epsilon_{U-B}$ & $N_{\text {obs }}$ & 2MASS & Remark $^{b}$ & $\mathrm{Sp}^{\mathrm{c}}$ \\
\hline 14846 & 164702.14 & -455143.9 & 20.941 & 16.078 & 4.848 & 3.436 & -2.737 & 0.022 & 0.021 & 0.030 & 0.109 & 0.127 & 31111 & $\ldots$ & & \\
\hline 14847 & 164702.15 & -454909.8 & 23.522 & 17.699 & 5.823 & $\ldots$ & $\ldots$ & 0.002 & 0.004 & 0.005 & $\ldots$ & $\ldots$ & 34300 & $16470214-4549097$ & & \\
\hline 14848 & 164702.15 & -454933.2 & 23.223 & 19.926 & 3.297 & $\ldots$ & $\cdots$ & 0.004 & 0.013 & 0.013 & $\ldots$ & $\ldots$ & 34300 & $\ldots$ & & \\
\hline 14849 & 164702.15 & -454846.3 & 23.760 & 20.594 & 3.165 & $\ldots$ & $\ldots$ & 0.009 & 0.012 & 0.015 & $\ldots$ & $\ldots$ & 33300 & $\ldots$ & & \\
\hline 14850 & 164702.15 & -455112.6 & 19.125 & 13.781 & 5.246 & 4.093 & -3.472 & 0.002 & 0.009 & 0.009 & 0.003 & 0.005 & 31132 & $16470215-4551126$ & $\mathrm{X}$ & O9.0Iab \\
\hline 14851 & 164702.15 & -455140.7 & 23.200 & $\ldots$ & $\ldots$ & $\ldots$ & $\ldots$ & 0.021 & $\ldots$ & $\ldots$ & $\ldots$ & $\ldots$ & 30000 & $\ldots$ & & \\
\hline 14852 & 164702.15 & -455211.6 & 22.094 & 19.502 & 2.592 & 2.065 & $\ldots$ & 0.013 & 0.008 & 0.015 & 0.014 & $\ldots$ & 34330 & $\ldots$ & & \\
\hline 14853 & 164702.15 & -455040.8 & $\ldots$ & 21.156 & $\ldots$ & $\ldots$ & $\cdots$ & $\ldots$ & 0.022 & $\ldots$ & $\ldots$ & $\cdots$ & 02000 & $\cdots$ & & \\
\hline 14854 & 164702.15 & -454651.6 & $\ldots$ & 21.711 & $\ldots$ & $\ldots$ & $\ldots$ & $\ldots$ & 0.055 & $\ldots$ & $\ldots$ & $\ldots$ & 03000 & $\cdots$ & & \\
\hline 14855 & 164702.16 & -454953.1 & 23.795 & 20.786 & 3.010 & $\ldots$ & $\ldots$ & 0.022 & 0.014 & 0.026 & $\cdots$ & $\ldots$ & 33300 & $\ldots$ & & \\
\hline 14856 & 164702.17 & -455154.4 & $\ldots$ & 20.884 & $\ldots$ & $\ldots$ & $\cdots$ & $\ldots$ & 0.041 & $\ldots$ & $\cdots$ & $\ldots$ & 03000 & $\ldots$ & & \\
\hline 14857 & 164702.17 & -455021.5 & 23.299 & 18.423 & 4.876 & $\ldots$ & $\cdots$ & 0.019 & 0.033 & 0.038 & $\ldots$ & $\ldots$ & 34300 & $\ldots$ & & \\
\hline 14858 & 164702.17 & -454721.3 & 17.277 & 15.644 & 1.624 & 1.293 & 0.703 & 0.006 & 0.008 & 0.010 & 0.006 & 0.003 & 31132 & $16470217-4547211$ & & \\
\hline 14859 & 164702.17 & -455107.6 & 22.693 & 19.586 & 3.107 & $\ldots$ & $\ldots$ & 0.002 & 0.092 & 0.092 & $\ldots$ & $\ldots$ & 31100 & $\ldots$ & & \\
\hline 14860 & 164702.17 & -455503.1 & 18.760 & 16.975 & 1.774 & 1.339 & 0.607 & 0.006 & 0.009 & 0.011 & 0.007 & 0.003 & 34332 & $\cdots$ & & \\
\hline 14861 & 164702.17 & -454944.6 & 18.646 & 16.817 & 1.820 & 1.448 & 0.830 & 0.001 & 0.003 & 0.003 & 0.006 & 0.006 & 34332 & $\cdots$ & & \\
\hline 14862 & 164702.18 & -455130.5 & 22.649 & 17.425 & 5.224 & $\ldots$ & $\ldots$ & 0.013 & 0.014 & 0.019 & $\ldots$ & $\ldots$ & 31100 & $\cdots$ & & \\
\hline
\end{tabular}

Notes.

a The negative numbered ID represents the calibrated data from Clark et al. (2005), Negueruela et al. (2010), and Piatti et al. (1998).

b X: X-ray emission stars; $\mathrm{x}$ : X-ray emission candidate.

c Spectral type-Clark \& Negueruela (2002, 2004); Clark et al. (2005); Crowther et al. (2006); Mengel \& Tacconi-Garman (2007); Negueruela et al. (2010); Clark et al. (2010); Dougherty et al. (2010).

(This table is available in its entirety in machine-readable and Virtual Observatory (VO) forms in the online journal with the recalibrated photometric data from Clark et al. (2005), Negueruela et al. (2010) and Piatti et al. (1998). A portion is shown here for guidance regarding its form and content.)

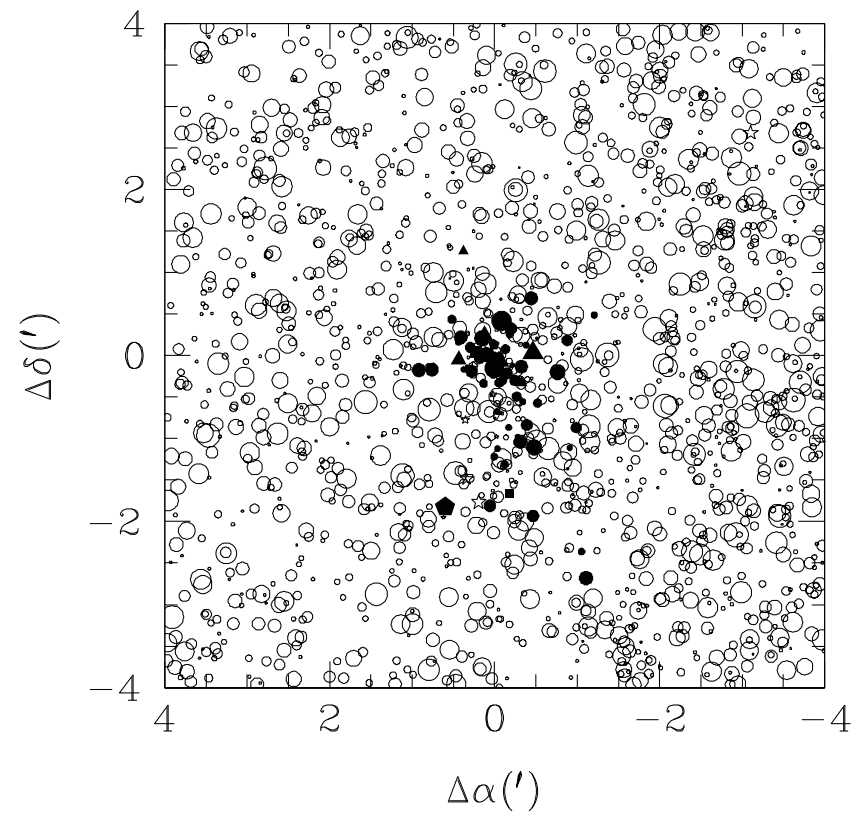

Figure 3. Finder chart for Westerlund 1. The filled symbols and star symbols represent the evolved stars (dot, OB supergiant; triangle, yellow hypergiant; square, red supergiant; pentagon, luminous blue variable; and star symbol, Wolf-Rayet star). The size of the symbols is proportional to the brightness, and only stars brighter than $V=20.5$ mag are shown. The position of the stars is given relative to the apparent center of the cluster $\left(\alpha=16^{\mathrm{h}} 47^{\mathrm{m}} 4^{\mathrm{s}} .1\right.$ $\left.\delta=-45^{\circ} 50{ }^{\prime} \cdot 65, \mathrm{~J} 2000\right)$

field population with X-ray emission. The statistical analysis in different wavelength regions gives the same radius as in the optical. It implies that our result may not be seriously affected by any environmental effects and that the radius derived in our analysis is reliable. We adopt 2.'5 as the radius of Westerlund 1. This value may be a lower limit. The physical size is $2.8 \mathrm{pc}$ if

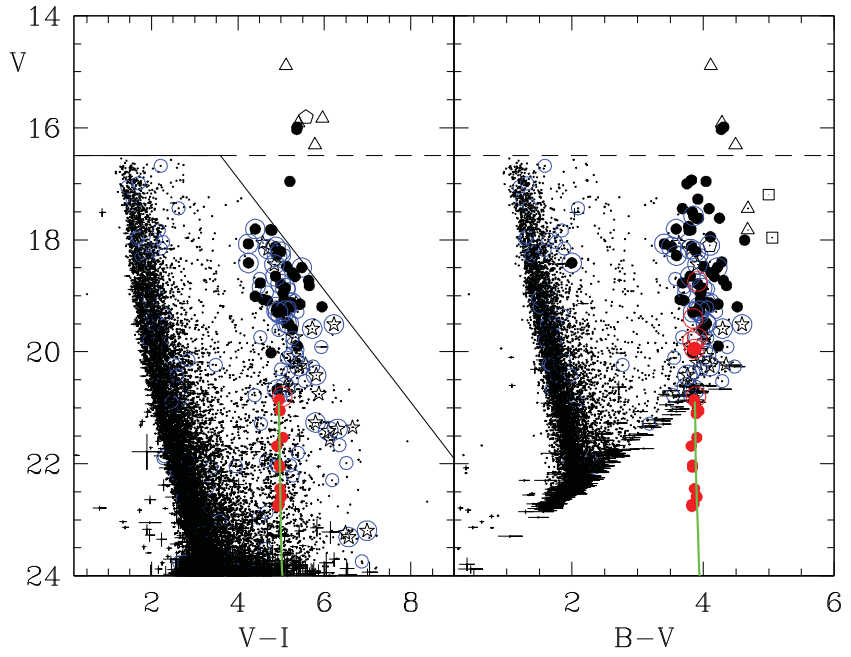

Figure 4. Color-magnitude diagrams of Westerlund 1. We present the saturation level in the $V$ band as a dashed-line, while the upper limit of the $V$ magnitude caused by the saturation level in the $I$ band is shown as a solid black line. A large dot, triangle, square, open pentagon, and star represent an OB supergiant, a yellow hypergiant, a red supergiant, a luminous blue variable, and a Wolf-Rayet star, respectively. A blue circle is in addition drawn around the known X-ray emission stars. We overplot the early-type stars (red filled circle) and blue supergiants (red open circle) of NGC 6231 as well as the zero-age main sequence relation (green solid line) by applying the reddening and distance of Westerlund 1, i.e., $\langle E(B-V)\rangle=4.19 \mathrm{mag}$ and $V_{0}-M_{V}=12.9 \mathrm{mag}$.

the distance to Westerlund 1 is $3.8 \mathrm{kpc}$ (see Section 3.3). The region outside this radius is assumed to be the field region.

In addition, we also calculated the half-mass radius of Westerlund 1 using the radial variation of the IMF (see Section 4) in the mass range of $5-85 M_{\odot}$. The half-mass radius of Westerlund 1 is $1.1 \mathrm{pc}$, which is $39 \%$ of the adopted radius. This value is consistent with the half-mass radii obtained by Brandner et al. (2008; $0.8 \mathrm{pc}$ for $m=10-32 M_{\odot}$ and $1.1 \mathrm{pc}$ for 


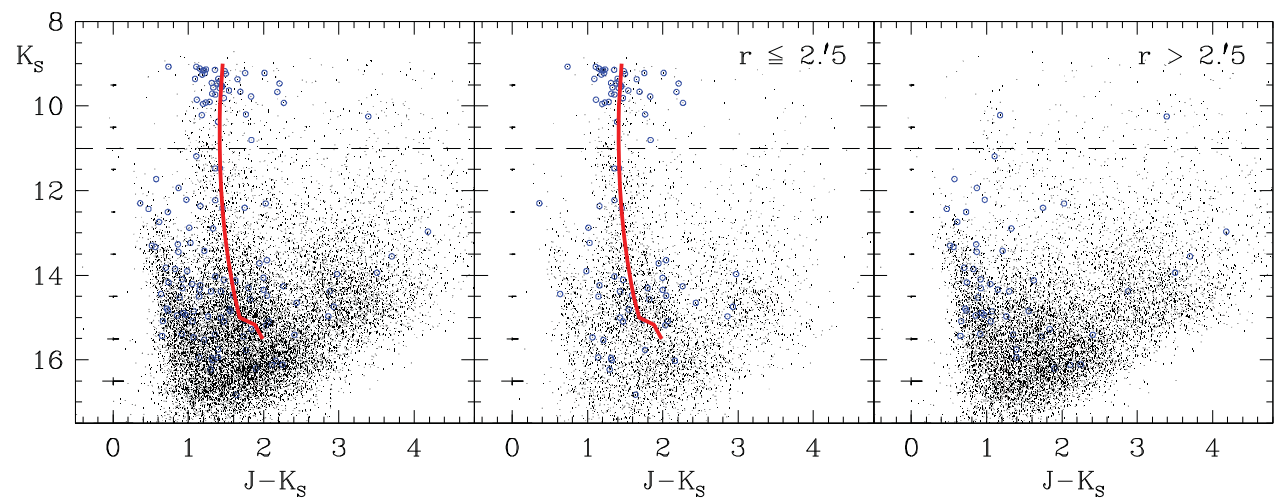

Figure 5. The $\left(K_{S}, J-K_{S}\right)$ color-magnitude diagrams for Westerlund 1 . The left, middle, and right panels show, respectively, all the stars, only the stars within 2 '.5 from the center to highlight the cluster sequence, and stars outside 2'.5. A dashed-line represents the linearity limit of the IRIS 2 detector. The mean photometric errors are shown at the left of all the diagrams. The empirical fiducial line adopted from the photometric data of several young open clusters is superimposed on the diagrams. The line is shifted for a reddening of $\left\langle E\left(J-K_{S}\right)\right\rangle=1.7$ mag and apparent distance modulus of $14.0 \mathrm{mag}$.

(A color version of this figure is available in the online journal.)

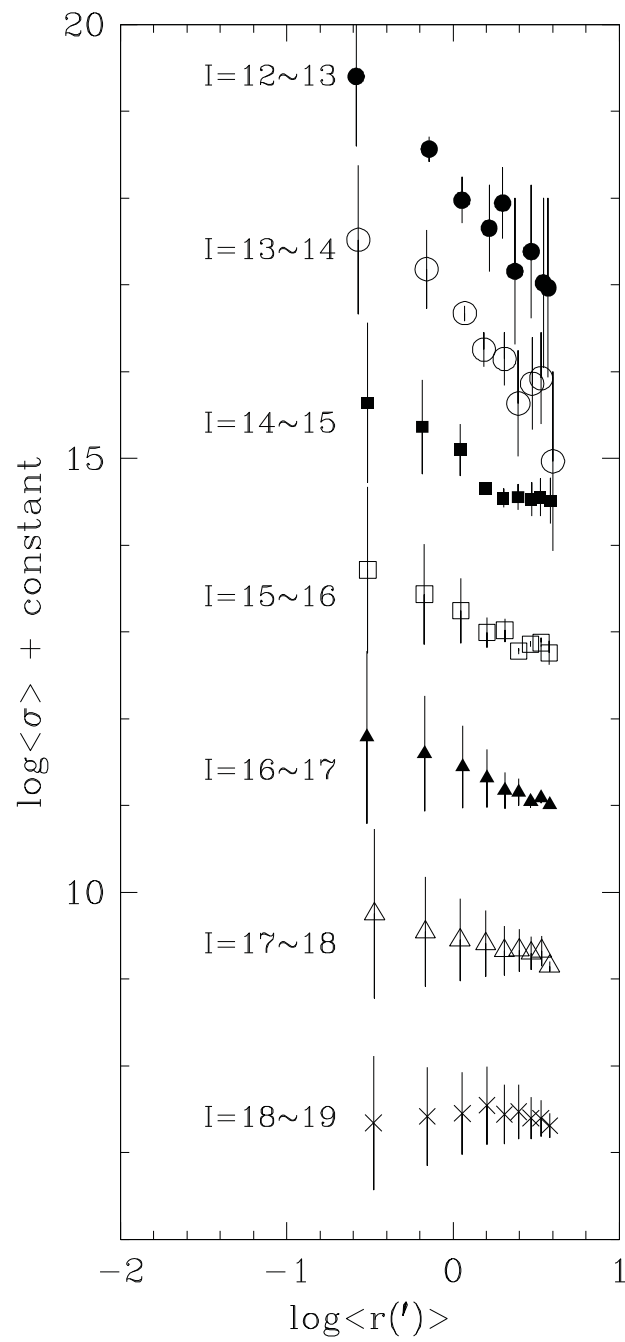

Figure 6. Surface density profiles of Westerlund 1 . The error at a given surface density is assumed to follow Poisson statistics. An arbitrary constant has been added to each density profile to separate the plots. The surface density of bright stars continues to decrease until the level reaches the surface density of field stars at a given brightness. The distance from the center to the point where both levels merge is the radius of Westerlund 1 . We obtain a radius of 2.5, equivalent to $2.8 \mathrm{pc}$, for a cluster distance of $3.8 \mathrm{kpc}$. $\left.m=3.5-10 M_{\odot}\right)$. According to their work, the half-mass radius of the low-mass group is larger than that of the high-mass group, and they attributed this to the presence of mass segregation. If we take into account the mass segregation and the unknown distribution of intermediate- to low-mass stars, the half-mass radius could be larger than the presented value. Hence, we also assign the value as a lower limit for the half-mass radius of Westerlund 1.

\subsection{Completeness Test}

Completeness of photometry is essential for statistical analyses, such as density profiles, luminosity functions, mass functions, etc. Therefore, a careful statistical treatment is required because photometric studies may be biased toward bright stars. Incompleteness is caused by crowding, bright nebulosity, spikes and bright wings of saturated stars, etc. For Westerlund 1, the main causes of incompleteness are crowding, the spikes of saturated stars, and bright wings, at longer wavelengths. Gennaro et al. (2011) presented the variation in the $K_{S}$ magnitude of $50 \%$ completeness across Westerlund 1 using a detailed twodimensional completeness map. In this paper, we check the completeness of our optical data.

In order to estimate the completeness of our optical data, we constructed a model cluster using a Monte Carlo method. The luminosity function of cluster stars, as well as foreground field stars, were derived in the $I$ band because the $I$ magnitude is less affected by reddening. Subsequently, the $V$ magnitude and $V-I$ color, with appropriate photometric errors, were generated from the $I$ magnitude by taking into account the appearance of the observational CMD. This allowed us to reproduce a realistic cluster. The observed radial surface density profiles as shown in Figure 6 were used to reproduce the crowding of the real cluster. In addition, we assumed the radial surface density profiles of faint stars to have the same profile as bright ones. On the other hand, field stars were uniformly generated across the whole area. A set of $V$ and $I$ images were made by adding these artificial stars to each sky image. Finally, we carried out PSF photometry, aperture correction, and standardization for the artificial stars in both the $V$ and $I$ images in the same way as done for the original data.

From the analysis of the model cluster, we estimated the completeness of our photometry. Figure 7 shows the completeness of photometry at different $V$ magnitude limits for several annuli. 


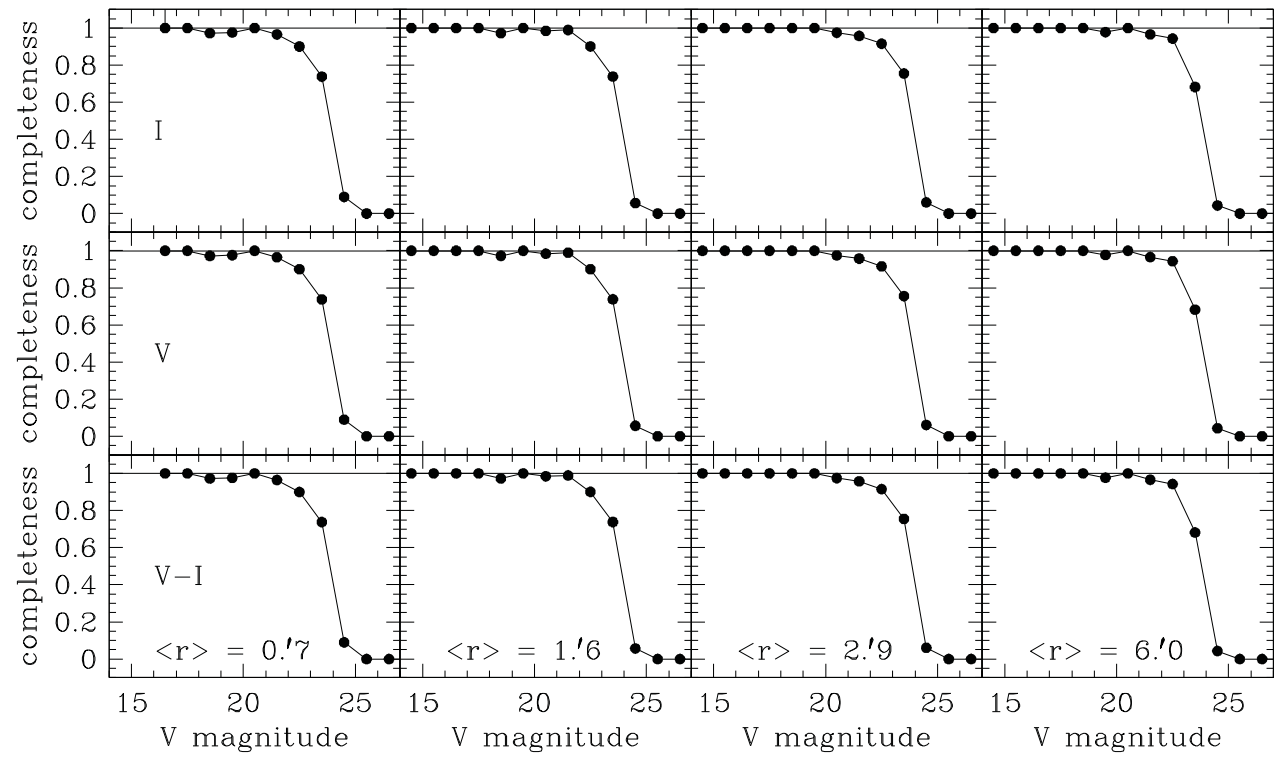

Figure 7. Completeness of the photometry against the $V$ magnitude for several radii. The completeness in the inner region is slightly lower than that of outer region. Our optical photometry seems to be about $90 \%$ complete down to $V \sim 22.0$ mag, even at the cluster center.

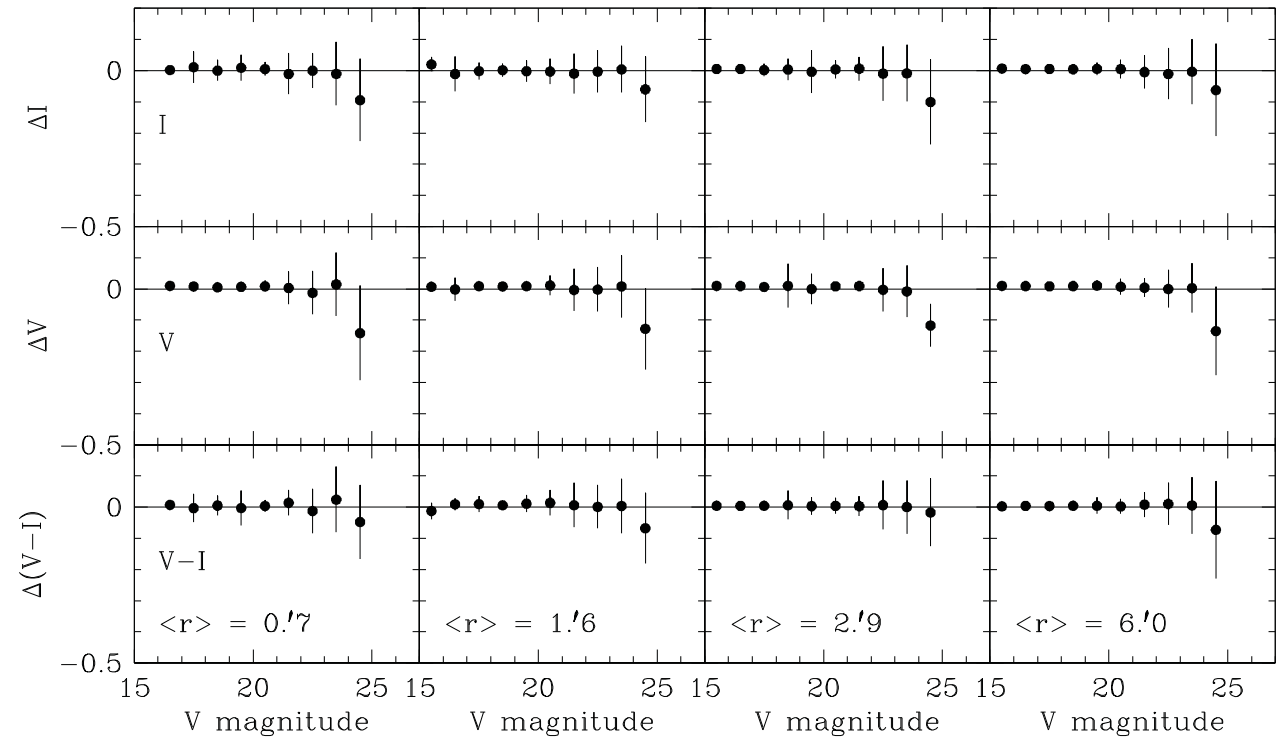

Figure 8. Difference between the input and output data for several radii. The output magnitudes are systematically brighter for faint stars ( $V \geqslant 23.0$ mag.), while well consistent with the input magnitudes for the others.

The radial variation of completeness is not as strong a function as that found by Gennaro et al. (2011). This is related to the observed passband. The effect of reddening is much more severe in the optical than in the NIR, and the difference in brightness between massive stars and low-mass stars is much larger in the optical; consequently, the $90 \%$ completeness level occurs at $V \sim 22 \mathrm{mag}$, equivalent to $\sim 20 M_{\odot}$, and may be an upper limit because faint stars could be expected to be much affected by the spikes and bright wings of saturated stars in long-exposure $I$-band images since we could not reproduce these spikes reliably. Moreover, quite a few stars in the $I$ band have no counterpart in the $B$ or $V$ bands because of the lower signal in $B$ or $V$ due to the severe reddening. Figure 8 represents the mean difference and the standard deviation between the input and output data, where $\Delta \equiv$ output - input. The data are well consistent with each other for bright stars, while the output data become systematically brighter for $V \geqslant 23 \mathrm{mag}$. This feature from PSF photometry using IRAF/DAOPHOT had already been reported in Sung et al. (1999). The fluctuations in the differences in the central region are slightly larger than those in the outer region due to the effect of crowding. From this analysis we conclude that our optical data are quantitatively and qualitatively complete down to 22 mag in $V$, even at the cluster center.

\subsection{Reddening and Distance}

In general, the reddening $E(B-V)$ of early-type stars is best determined from the $(U-B, B-V)$ diagram. Unfortunately, as we could not obtain a reliable $U-B$ color index even for the brightest star due to the red leak of the SDSS $u$ filter (see Appendix A for details), as well as to the very high reddening, a more fundamental, but less reliable method, namely, the spectral type versus intrinsic color relation, was used to determine the reddening. Although the observation was made only for longer 


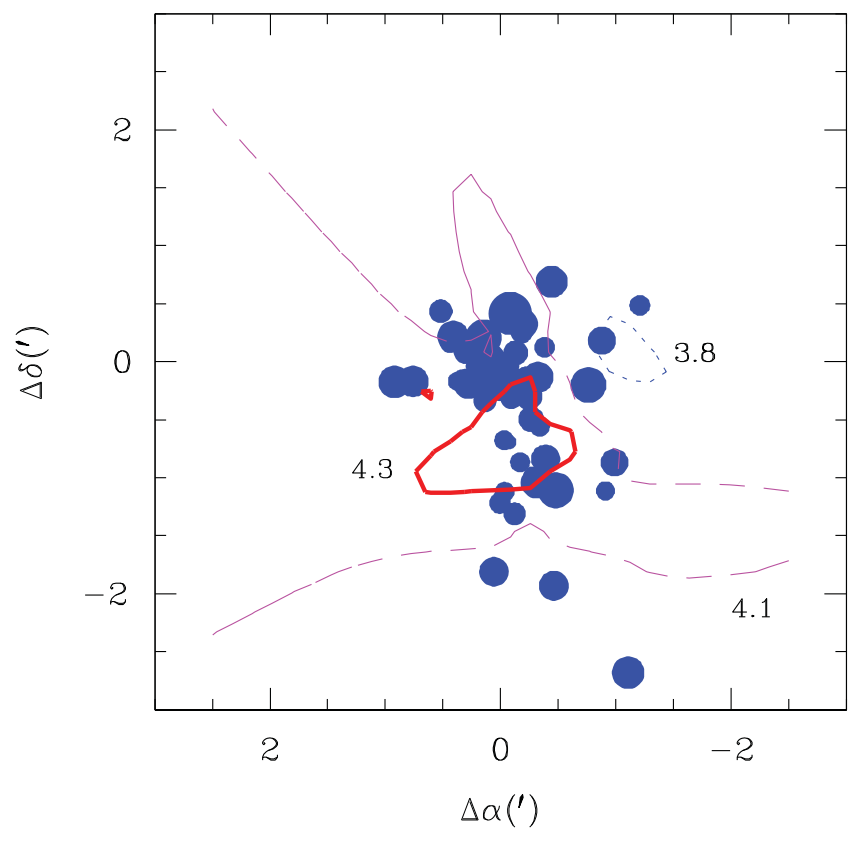

Figure 9. Reddening map of Westerlund 1. The blue dots represent OB type supergiants, the size of the dots are proportional to the brightness. The red solid line, dashed magenta line, and dotted blue line represent contours of $E(B-V)=4.3,4.1$, and 3.8 , respectively. The reddening map indicates that the innermost region is the most heavily reddened.

(A color version of this figure is available in the online journal.)

wavelengths $(\lambda>5500 \AA)$ to achieve a reasonable signalto-noise ratio, the spectral classification of Negueruela et al. (2010) for OBSGs is the most recent result with a $8.0 \mathrm{~m}$ class telescope, and was helpful for this work. To determine the color excess of individual stars, we adopted the calibration of Flower (1977), which provides the intrinsic $B-V$ color, effective temperature, and bolometric correction for various luminosity classes. The intrinsic $B-V$ color of 53 OBSGs was determined by interpolating the spectral type versus intrinsic relation, and then calculating the reddening $E(B-V)$. We obtained the mean value of $\langle E(B-V)\rangle=4.19 \pm 0.23$ (s.d.) mag. Assuming $R_{V}=3.1$, the total mean extinction $\left(A_{V}\right)$ is about 13.0 mag. If we simply assume the intrinsic value of $B-V=-0.25 \mathrm{mag}$ and $V-I=-0.28 \mathrm{mag}$ for OBSGs, the ratio of color excess is $\langle E(V-I)\rangle /\langle E(B-V)\rangle=1.26$. This implies that the reddening law toward Westerlund 1 is fairly normal, i.e., $R_{V} \sim 3.1$.

We also constructed the reddening map and present it in Figure 9. In the figure, the large dots denote the position of 53 OBSGs. And solid red, dashed magenta, and dotted blue lines represent contours of $E(B-V)=4.3,4.1$, and 3.8, respectively. The size of the dots is proportional to the brightness of individual stars. The reddening is the highest near the center and shows a gradient from east to west. In general, we could expect a cavity of extinction near the cluster center as is seen in NGC 3603 (Sung \& Bessell 2004) due to the strong stellar wind from massive stars. Contrary to our expectations, the reddening was highest near the cluster center. The region is coincident with the extended radio emission source A2 of Dougherty et al. (2010) and the $8 \mu \mathrm{m}$ Spitzer IRAC emission feature (see Figure 4 of Dougherty et al. 2010). According to Dougherty et al. (2010), the extended radio emission source A2 may be associated with the WN7 star WR B. These facts imply that outflowing material from WR stars is forming dust around stars and is evidenced from the reddening map.
Given photometric differences among previous studies, the results are in good agreement with each other within the errors, e.g., $E(B-V)=4.2 \pm 0.4$ (Negueruela et al. 2010) and $E(B-V)=4.3 \pm 0.2$ (Piatti et al. 1998). Negueruela et al. (2010) discussed the large deviation of their average color excess ratio $\langle E(V-I)\rangle /\langle E(B-V)\rangle$ from the standard value (Rieke $\&$ Lebofsky 1985). They attributed the deviation to the error in their $B$-band photometry, putatively the zero point in the band. However, since their photometric data were transformed to the standard $R_{C} I_{C}$ system of Landolt (1992), the color excess ratio should have been compared with the standard reddening law in the Johnson-Cousins photometric system, not in the Johnson's $R I$ system. Given the error of the color excess ratio of Negueruela et al. (2010), their result seems to be close to the standard reddening law.

The distance to open clusters is, in general, determined by fitting the ZAMS relation to the MS of the cluster. However Westerlund 1 is so obscured that it is nearly impossible to define the MS of Westerlund 1 in the $(V, V-I)$ CMD. Although the location of MS stars in the CMD is well defined, the color range of early-type stars is very narrow, and the MS stars appear to be almost perpendicular to the color axis in the CMD. And therefore it would give a less reliable distance to the cluster if the ZAMS relation were employed. Piatti et al. (1998) seem to be confronted with this difficulty. They determined a distance to Westerlund 1 of 1.5-2.0 kpc by matching the absolute magnitudes of supergiants to the isochrone of age 4.0 Myr. However, given the uncertainties in the reddening correction of individual stars and the intrinsic scatter of evolved stars (see Figure 3 in Humphreys \& Davidson 1979), it seems that the distance obtained by Piatti et al. (1998) is less reliable. Indeed the value is substantially different from those of other estimates, e.g., $5.0 \mathrm{kpc}$ (Clark et al. 2005; Negueruela et al. 2010; Crowther et al. 2006), $3.9 \mathrm{kpc}$ (Kothes \& Dougherty 2007), $4.0 \mathrm{kpc}$ (Gennaro et al. 2011; Koumpia \& Bonanos 2012), and $3.7 \mathrm{kpc}$ (Koumpia \& Bonanos 2012).

For this reason, the use of NIR photometric data, which are less affected by interstellar extinction, is inevitable in determining the distance to Westerlund 1. As shown in both Brandner et al. (2008) and Gennaro et al. (2011), the appearance of the turn-on point from the PMS to the MS (they called it the transition zone) provides a fiducial marker for the empirical fitting method and encourages us to construct an empirical relation using photometric data of other clusters. Mayne et al. (2007) have constructed empirical isochrones in the $(V, V-I)$ diagram to determine fundamental parameters of young stellar associations and clusters. Since the turn-on position is sensitive to the age, we had to select clusters with almost the same age as Westerlund 1, 5.0 Myr. The young open clusters NGC 2362 (3.0-6.0 Myr, Moitinho et al. 2001), NGC 6231 (2.5-4.0 Myr, Sung et al. 1998; 3.0-5.0 Myr, Baume et al. 1999; 4.5 Myr, Santos \& Bica 1993), and NGC 6823 (2.0-7.0 Myr, Bica et al. 2008) were selected under this condition. The empirical fiducial line (EFL) constructed in the $\left[M_{K_{S}},\left(J-K_{S}\right)_{0}\right]$ plane is presented in Figure 5 after correcting for the reddening and the difference in distance. The EFL is very useful because it is independent of stellar evolution models as well as being less affected by variations in metallicity. Details on the EFL are addressed in Appendix B. We brightened the $\left(K_{S}, J-K_{S}\right)$ CMD of Westerlund 1 by an apparent distance modulus of 14.0 mag to fit it to the turn-on point from the PMS to the MS of the EFL by eye and compared the observed color indices with those of EFL for stars with $1<J-K_{S}<2$ and 
$11<K_{S}<13$. The mean color excess of $\left\langle E\left(J-K_{S}\right)\right\rangle=$ $1.70 \pm 0.21$ (s.d.) was thus obtained. In order to compute the total extinction in $K_{S}$, i.e., $A_{K_{S}}$, Equation (2) of Kim et al. (2006) was used, where we adopted the exponent of $\alpha=1.66$ according to their suggestion. The reddening law results in $A_{K_{S}}=1.12 \pm 0.14 \mathrm{mag}$. Consequently, the apparent distance modulus subtracted by $A_{K_{S}}$ is $K_{S 0}-M_{K_{S}}=12.9 \mathrm{mag}$, equivalent to $3.8 \mathrm{kpc}$. Our result is consistent with the three previous studies (Kothes \& Dougherty 2007; Gennaro et al. 2011; Koumpia \& Bonanos 2012).

The distance to Westerlund 1 is still being debated in the literature. Clark et al. (2005), Negueruela et al. (2010), and Crowther et al. (2006) prefer a distance of $5 \mathrm{kpc}$, which places Westerlund 1 in the Norma arm. On the other hand, a distance of $1.1 \mathrm{kpc}$ adopted by Piatti et al. (1998) corresponds to the Sagittarius arm. To constrain the distance of the cluster we referred to the NIR CMD of control field in Figure 4 (upper right panel) of Gennaro et al. (2011) and assumed that the Sagittarius arm, Scutum-Centaurus arm, and Norma arm to be at $1.1 \pm 0.4 \mathrm{kpc}, 3.0 \pm 1.0 \mathrm{kpc}$, and $5.4 \pm 0.8 \mathrm{kpc}$ toward Westerlund $1(l=339.5, b=-0.4)$, respectively. If Westerlund 1 is in the Sagittarius arm, the bluest sequence in Figure 4 of Gennaro et al. (2011) should penetrate the sequence of Westerlund 1 or slightly overlap it because they are a cospatial disk population. As there is an obvious gap below the MS turn-on of Westerlund 1 (see also the upper left panel in their figure), the distance derivations of Westerlund (1961), Borgman et al. (1970), and Piatti et al. (1998) should be ruled out. It can be assumed that the group of stars at $K_{S} \sim 14 \mathrm{mag}$ and $J-K_{S} \sim 0.8$ are the representative field star population of the spiral arm and have the same photometric properties in all three arms, and that the effects of reddening within a single spiral arm are negligible. The bright stars, which are likely to belong to the Sagittarius arm, will be at $K_{S} \sim 17.3 \mathrm{mag}$ and $J-K_{S} \sim$ 2.5 if those are reddened and dimmed by the reddening of Westerlund $1\left[E\left(J-K_{S}\right)=1.7 \mathrm{mag}\right.$ and $\left.A_{K s}=1.1 \mathrm{mag}\right]$ and the difference in distance moduli $\left[\Delta\left(K_{S 0}-M_{K_{S}}\right)=2.2 \mathrm{mag}\right]$ between the Sagittarius arm and Scutum-Centaurus arm. This approach allows us to explain the presence of a population with $2 \lesssim J-K_{S} \lesssim 3$.

On the other hand, the bright stars would be shifted to $K_{S} \sim 18.6$ mag were the stars in the Norma arm, i.e., $\Delta\left(K_{S 0}-M_{K_{S}}\right)=3.5 \mathrm{mag}$ instead of $2.2 \mathrm{mag}$. Were Westerlund 1 in the Norma arm, the field star population in the arm should have barely been detected because it would approach the detection limit of $K_{S}$. See Figure 4 (upper right panel) of Gennaro et al. (2011) again, the population of field giants is obliquely stretched over a wide color range $\left(1 \lesssim J-K_{S} \lesssim 5\right)$. There seems to be a void near $K_{S} \sim 12 \mathrm{mag}$ and $J-K_{S} \sim 2.2 \mathrm{mag}$ in the sequence. If this feature is real, it implies that there is an interarm region beyond the Sagittarius arm in the line of sight, and that the redder sequence of field giants belongs to the second spiral arm from the Sun toward Westerlund 1. In this way, the stars with $J-K_{S} \geqslant 2.0$ in the CMD are likely to be a population in the Scutum-Centaurus arm. In conclusion, we believe we can well constrain the location of Westerlund 1 to the Scutum-Centaurus arm, which is consistent with our result, and make a thread of connection between our interpretation and the results of Kothes \& Dougherty (2007).

\subsection{Age of Westerlund 1}

The widely used method for estimating the age of an open cluster is to fit the theoretical isochrone to the MS turnoff in the CMDs or the Hertzsprung-Russell diagram (HRD), by assuming that the members are coeval. For the case of Westerlund 1, since the MS turnoff is very faint due to severe extinction and the MS turn-on is too faint to be detected from optical photometry it is nearly impossible to directly apply this method to the optical CMDs. For this reason, previous studies have constrained the age of Westerlund 1 to be 3-5 Myr using the lifetime of massive evolved stars (Clark et al. 2005; Crowther et al. 2006), isochrone fitting to the evolved stars (Negueruela et al. 2010), or the PMS-MS transition zone in the NIR CMD (Brandner et al. 2008; Gennaro et al. 2011). Recently Kudryavtseva et al. (2012) have conducted a Bayesian analysis using kinematic members. In the case of Negueruela et al. (2010), their adopted isochrone seems not to be the best prediction for the positions of evolved stars in the HRD, especially $O$ type supergiants. Since the difference in the temperature scales between Martins et al. (2005) and Flower (1977) for late-O type supergiants is not very significant, we attribute their results to the incorrect estimate of the distance to Westerlund 1. Sung et al. $(1997,2000)$ found a discrepancy in the age and mass of PMS stars among PMS evolution models. Negueruela et al. (2010) also pointed out a younger age of Westerlund 1 inferred from PMS model (Brandner et al. 2008). It implies that the evolution models of PMS stars still have some limitations, e.g., the introduction and handling of mass accretion in PMS evolution models (Hosokawa et al. 2011). Kudryavtseva et al. (2012) did not take into account the comprehensive member selection for RSGs and X-ray-emitting PMS stars, whose membership is very important to draw the formation picture of Westerlund 1. We would like to suggest that more careful member selection may be needed in their work. In this paper, for the evolved stars, we will use new theoretical stellar evolution models with/without rotation (Ekström et al. 2012) and $Z=0.014$ to estimate the age of Westerlund 1 in the same manner as Negueruela et al. (2010) and compare the result with that of previous studies.

We applied the total extinction inferred from OBSGs and the distance modulus of 12.9 mag to the 62 evolved stars in Westerlund 1. The spectral type of individual stars was transformed to the effective temperature using the calibration of Flower (1977). The observational HRD of the evolved stars is shown in Figure 10. Finally, four isochrones of different ages are superimposed on the diagrams. The 4.0 Myr isochrone (blue solid line) without rotation gives the best fit to the evolved stars in the left panel of Figure 10. The model well predicts the position of YHGs in the HRD; however, the predicted position of OBSGs is slightly cooler than observed. Most of the observed stars are fainter and cooler than predicted, when the 3.0 Myr isochrone (upper dashed line) is placed on the HRD. The age of 3.5 Myr seems to be matched well to our data. Indeed, this age is the same as that estimated from the stellar evolution models of Schaller et al. (1992). On the other hand, the stellar models with rotation seem to better predict the position of observed stars in the HRD (see the right panel of Figure 10). The 5.0 Myr isochrone (blue solid line) traces not only the locus of the OBSGs but also those of the two late-B supergiants and five bright YHGs. Most of the massive stars are confined between the 4.0 Myr and 6.0 Myr isochrones, respectively. Although the high binary fraction of $\geqslant 62 \%$ (Crowther et al. 2006), variability (Bonanos 2007; Clark et al. 2010), and uncertainties in the spectral classification (Negueruela et al. 2010) may affect the location of the evolved stars in the HRD, we assign the age of Westerlund 1 to be $5.0 \pm 1.0 \mathrm{Myr}$, if we 


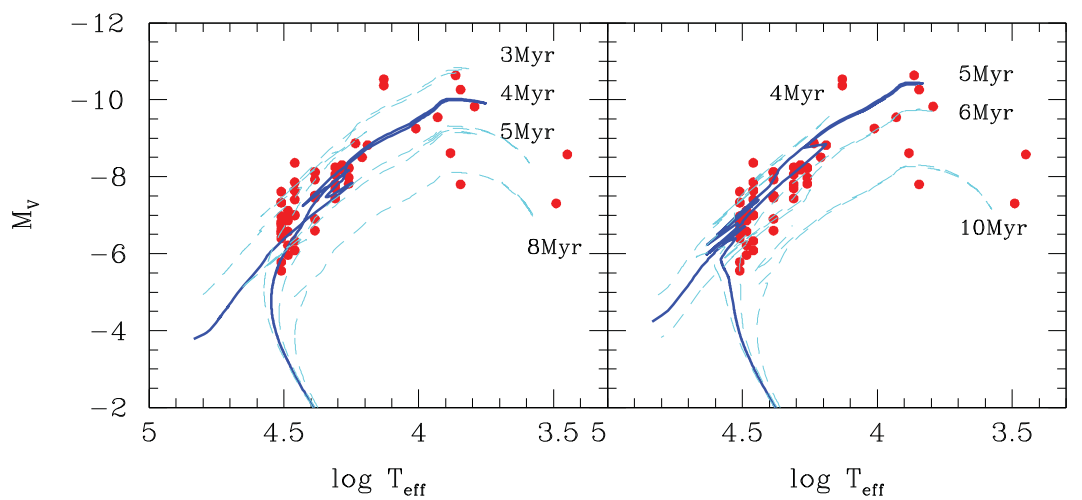

Figure 10. Age of Westerlund 1. The left panel shows the loci of stellar evolution models without rotation and the right panel the loci of models with rotation. Each red dot represents 62 evolved stars, and four isochrones with different age are superimposed on each diagram. The solid blue line denotes the best-fit model. The stellar evolution models without rotation give a slightly younger age than those with rotation. The latter models seem to reproduce very well the positions of the evolved stars in the Hertzsprung-Russell diagram.

(A color version of this figure is available in the online journal.)

assume a near coeval star formation (see also Kudryavtseva et al. 2012).

Our result of $5.0 \pm 1.0 \mathrm{Myr}$ from NIR data is compatible with the results of Clark et al. (2005), Crowther et al. (2006), and Negueruela et al. (2010), which discussed the evolutionary timescale of massive stars, and with that of Kudryavtseva et al. (2012) based on a Bayesian analysis as well as with that of Gennaro et al. (2011) based on the isochrone fitting. It is also consistent with the formerly assumed age of Westerlund 1 (3-6 Myr) in Section 3.3. However, the presence of RSGs and relatively faint YHGs makes us concerned as to whether or not the population within Westerlund 1 is coeval. If these two groups of stars are real members, current evolution models indicate that the age of Westerlund 1 should be extended to 8.0-10.0 Myr as shown in Figure 10. More discussions will be presented in Section 6.1 .

\section{THE INITIAL MASS FUNCTION}

Using the relation between the spectral type and bolometric correction of Flower (1977), the absolute magnitude of the evolved stars were converted to the absolute bolometric magnitude. Next, we constructed a mass-luminosity relation, especially for the stars at the post-MS stage, based on the probability distribution of a given mass along the evolutionary track. We directly interpolated the stellar evolution models and determined the masses of individual stars from their positions in the HRD. However, the masses of LBVs and WRs still remain uncertain because the intrinsic properties of these stars are still not well known. Crowther et al. (2006) inferred that the initial masses of WRs in Westerlund 1 is in the range of $40-55 M_{\odot}$ from the evolutionary models. Assuming the Kroupa IMF, a simple simulation was conducted to estimate the mass range of WRs using the stellar evolution models (Ekström et al. 2012). The masses of model $\mathrm{WN}$ and $\mathrm{WC}$ stars are distributed in the range of 50-70 $M_{\odot}$ and $40-70 M_{\odot}$, respectively. Since it is impossible to determine the exact initial mass of individual WRs from the current data and current calibrations, all WR stars were included in the highest mass bin. On the other hand, according to Massey (2003), only the most massive stars with an initial mass exceeding $85 M_{\odot}$ could evolve to become LBVs. The masses of the two LBVs (W9 and W243) in Westerlund 1 may be at least $85 M_{\odot}$. The two LBVs were also included in the highest mass bin. For the rest of the stars, which are presumably MS or stars near the MS turnoff, i.e., those with $V-I \geqslant 4$ and $B-V \geqslant 3$ and which lie within the radius of 2.5 , the $V$ magnitude was converted to absolute magnitude using the reddening map in Figure 9 and a distance modulus of 12.9 mag. For these stars, the conversion from color indices to the effective temperature is much more uncertain unless their spectral type or individual reddening is available, and therefore the mass-absolute-magnitude relation of the 5.0 Myr isochrone with rotation was used in order to determine their masses. We also determined the mass of field interlopers, with the same photometric properties as Westerlund 1, to subtract the contribution of field stars in the mass function, after correcting for the mean extinction and distance modulus. Note that two YHGs (W12a and W265), four RSGs (W20, W26, W75, and W237), two OBSGs (W228b and W373), and four WRs (N, T, X, and S) have been excluded in the mass function calculation because the membership of these stars is uncertain, and because these OB and WR stars are outside the adopted radius of Westerlund $1\left(r_{\mathrm{cl}}=2 \cdot 5\right)$. Recent simulations on the dynamical evolution of young open clusters (Allison et al. 2010) suggested the existence of ejected stars due to the interaction between massive stars. In addition, as Westerlund 1 may have experienced a number of SNe, we could expect a number of runaway stars around Westerlund 1. We therefore cannot exclude the possibility that these massive stars may be ejected members of Westerlund 1. The membership of these stars is left for future study.

To calculate the mass function $\xi$ ( $\equiv N / \Delta \log m /$ area), we counted, respectively, the number of stars within the adopted radius of Westerlund 1 and field region $\left(r>2 .{ }^{\prime} 5\right)$ for a given mass bin $(\Delta \log m=0.2)$. The latter was normalized by multiplying the areal ratio of cluster to field. This therefore represents the probable number density of field interlopers within the cluster region. The total number density of stars within each mass bin in the cluster region was subsequently adjusted by subtracting the number of interlopers in the same mass bin. A complementary IMF for the stars with intermediate mass was also derived from NIR photometry. As the model provides $M_{V}$ and $(B-V)_{0}$, the absolute $V$ magnitude of the $5 \mathrm{Myr}$ isochrone with rotation, was converted into the absolute $K_{S}$ magnitude using the $\left(V-K_{S}, B-V\right)$ relation calculated by one of the authors (M.S.B.). The transformed $M_{K_{S}}$ was dimmed by the apparent distance modulus of 14 mag and transformed to mass for a given star, by interpolating the observed $K_{S}$ magnitude into the mass-luminosity relation, where only MS 


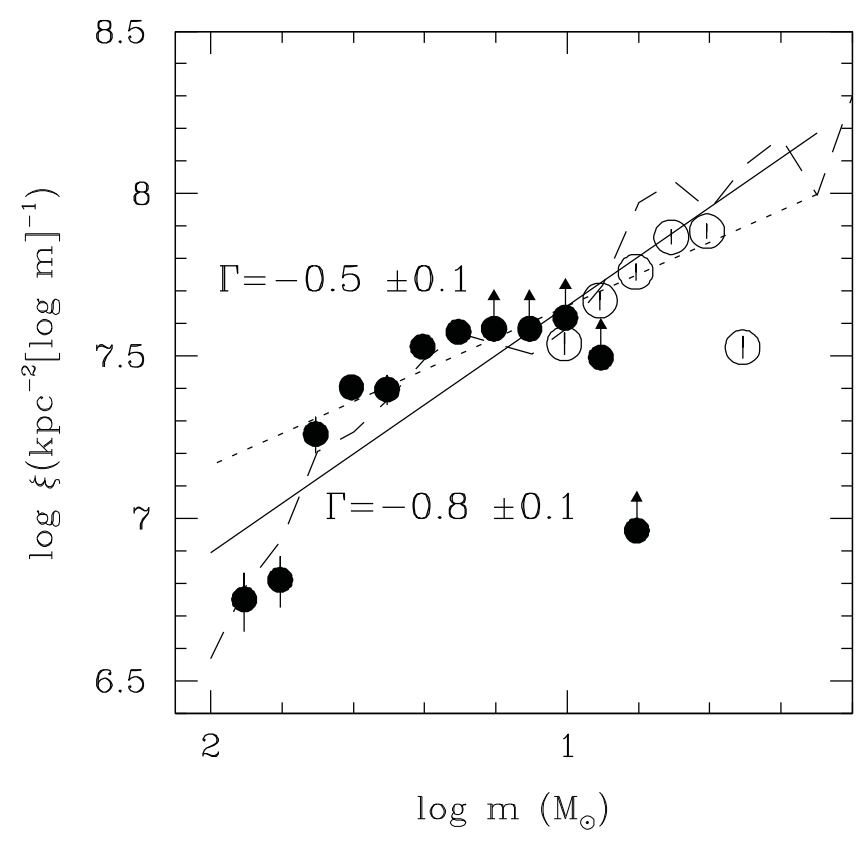

Figure 11. Initial mass function of Westerlund 1. The dots and circles represent the initial mass function derived from optical photometry and near-infrared photometry, respectively. The dashed line denotes the scaled initial mass function of NGC 3603 (Sung \& Bessell 2004). We obtain a shallow slope of $\Gamma=-0.8 \pm 0.1$ in the mass range of $\log m=0.7-2.0$. The shape of the IMF of NGC 3603 and Westerlund 1 are very similar.

stars that were not affected by the nonlinearity of the NIR detector were used. The stars away from the cluster region were also used to estimate the contribution to the mass function by field interlopers. A rather reliable IMF in the low-mass regime was obtained by Gennaro et al. (2011). We present the IMF of Westerlund 1 in Figure 11. To avoid effects of binning, an additional mass function was calculated by shifting the mass bin by 0.1 in logarithmic mass scale. The large dots and open circles represents the IMFs derived from optical data and NIR data, respectively. The error is assumed to follow Poisson statistics. According to the completeness of our optical photometry, the reliable mass limit from the optical data is about $20 M_{\odot}$, and the IMF below this limit may be a lower limit. The shape of the IMF is very similar to that of NGC 3603 (the dashed line in the figure). The IMF revealed a shallow slope $(\Gamma=-0.8 \pm 0.1)$, when we computed the slope in the mass range of $\log m=0.7$ to $\log m=2$. If we exclude the two highest mass bins because of the loss of massive stars through evolution, the slope of the IMF is much shallower $(\Gamma=-0.5)$. Although there are some uncertainties in the IMF derived from NIR photometry, such as the differential reddening, incompleteness, and subtraction of field interlopers, the slope is in good agreement with the results of Sung \& Bessell (2004) and Stolte et al. (2006; $\Gamma=-0.9)$ for NGC 3603. The shape and slope of the IMF, $\Gamma=-0.7 \pm 0.1$, did not vary significantly when we included the excluded stars mentioned above (two YHGs, four RSGs, two OBSGs, and four WRs). More details about the shape of the IMF will be discussed in Section 6.2.

Observational evidence of a large number of low-mass stars present in starburst open clusters has been reported. Based on this evidence, we computed the total mass of Westerlund 1 assuming the IMF of the intermediate- to low-mass regime $\left(10 M_{\odot}>m>0.08 M_{\odot}\right)$ to be that of NGC 2264 (Sung \& Bessell 2010) and that of Kroupa (2002), respectively. After appropriately scaling the level of the two IMFs to ours and multiplying by the surface area and the mass bin, the IMFs in the massive to low-mass regime were integrated. The total mass of Westerlund 1 is about $7.3 \times 10^{4} M_{\odot}$ from the IMF (NGC 2264 plus ours) and about $7.8 \times 10^{4} M_{\odot}$ from that of Kroupa plus ours, respectively. These are well consistent with the dynamical mass of Mengel \& Tacconi-Garman (2007) within their uncertainty. The total mass derived by Gennaro et al. (2011) would be comparable to ours had they adopted a rather flat slope in the massive part of the IMF, instead of a single overall slope.

Another way to estimate the total mass of Westerlund 1 is to use the information on the radial variation of the IMF in Figure 12 (see Section 5 for details). Due to a lack of information on the radial distribution of intermediate- to low-mass stars, a single slope of the IMF extrapolated to $5 M_{\odot}$ was assumed within a given annulus. The sum of masses derived within five annuli yielded a total mass of $5.1 \times 10^{4} M_{\odot}$. This value is for masses greater than $5 M_{\odot}$, and therefore is a lower limit to the total mass of Westerlund 1. Again, we confirm that Westerlund 1 is the most massive open cluster in the Galaxy by comparing the total mass of other starburst open clusters (see Table 4 in Clark et al. 2005). Hence, the mass of Westerlund 1 is comparable to that of globular clusters in the Galaxy, and the study of Westerlund 1 could give hints to the formation process of globular clusters in the early universe.

\section{MASS SEGREGATION}

Observational evidence of mass segregation in open clusters has been confirmed from the radial variations of mean mass, the ratio of high mass to low mass, the slope of the IMF, and minimal spanning tree method. In this paper, we investigated the mass segregation using the radial variation of the IMF of bright evolved stars. From the masses of individual stars determined above, the IMFs were computed for different annuli of width of 0.5. The IMF for a given annulus was adjusted for the contribution of field interlopers. The slopes of the IMF were computed only for $\log m \geqslant 1.4$. We present the result in Figure 12 (left panel).

Clearly, the IMF of the innermost region shows the shallowest slope. The slopes gradually steepen with increasing distance from the center, however there is an abrupt change at the fourth ring $(r=1$ '.7). If this feature is not related to a statistical fluctuation, it may imply a substructure in Westerlund 1. Unlike Gennaro et al. (2011), we simply assumed a circular shape for the cluster. The departure from circularity could cause a distorted surface density profile. Although an apparent elongation is evident within $r=1.4$, the slopes of the IMF become systematically steeper within the annulus. We also performed the same approach as above by taking into account the elongation using the same parameters as in Gennaro et al. (2011), $a: b=3: 2$ and a tilt angle $\sim 22.5$ (see Figure 12, right panel). The semimajor axis is increased by 0.5 . The slopes of the IMF were systematically steeper $(\Gamma=-1.3,-1.6,-1.7$, and -3.2 ) as the central distance increases within $a=2.0$, while a relatively flat slope $(\Gamma=-1.8)$ was found between $a=2^{\prime}$.0 and $a=2 ' .5$. Although the discontinuity in the slopes occurs at a slightly different position with the different approaches, our result suggests the presence of a halo structure in Westerlund 1. To check whether or not a similar pattern could be found in the surface density profiles, we drew surface density profiles for several cutoff magnitude in Figure 13. A slight change in the slope could be seen at $\log r \sim 0.2$, equivalent to 1 '.6 in the surface density profiles. Since the same trend could be found in 

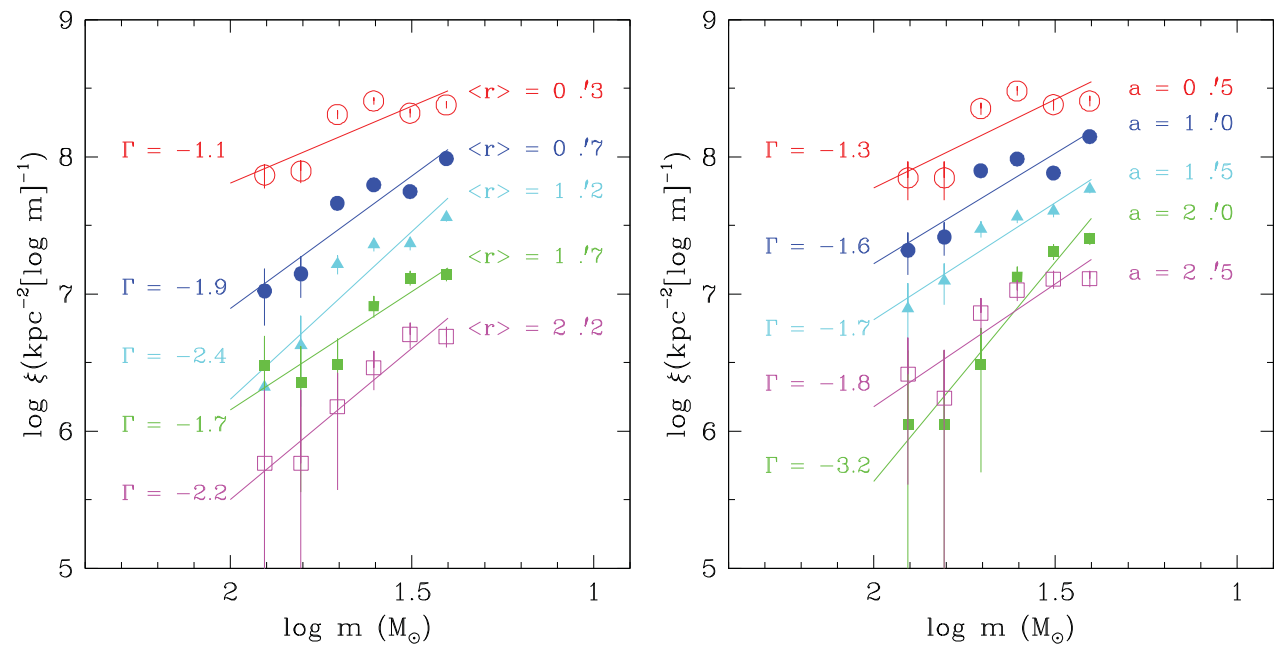

Figure 12. Radial variation of the IMF. We present the IMFs measured at increasing distance from the cluster center from top to bottom in the diagrams. Left: circular fitting; right: ellipse fitting. The slopes become systematically steeper inside $r \sim 1$ 1.7 $^{\prime} a \sim 2 .^{\prime} 0$ where an abrupt change in slope occurs. See the main text for details. (A color version of this figure is available in the online journal.)

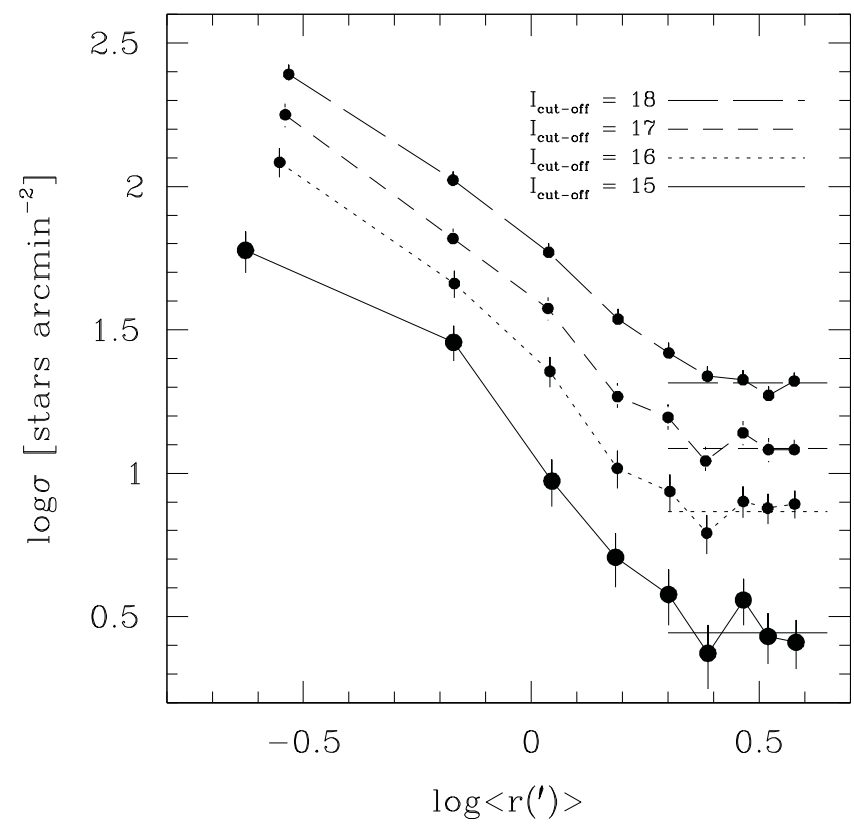

Figure 13. Surface density profiles of Westerlund 1 with several cutoff I magnitude. The horizontal line represents the average surface density of the field region for a given cutoff magnitude. As the completeness of photometry for $I<18 \mathrm{mag}$ is over $80 \%$ even in the cluster center, the incompleteness of data is not corrected

all profiles with different cutoff $I$ magnitudes, the change in slope may not be a consequence of data incompleteness. If the abrupt change in the radial variation of the IMFs is related to that of the surface density profile, it may also imply that Westerlund 1 has substructure (a core and a halo). Although we could not confirm the existence of a substructure in Westerlund 1, exploring the presence of a halo based on reliable membership criteria will be one of the important challenges to understand the dynamical evolution of massive open clusters. Kook et al. (2010) found a halo in the surface density profile of a compact young cluster Hogg 15.

On the other hand, there may be another interpretation for the fluctuation of the slope of the IMF within a certain radius, namely, limited mass segregation. Mass segregation was only found in the inner region, while massive stars in the outer region were well mixed. According to Raboud \& Mermilliod (1998) and Hillenbrand \& Hartmann (1998), mass segregation occurs only for massive stars in NGC 6231 and the Orion Nebula Cluster. However, Gennaro et al. (2011) found mass segregation even for the low-mass stars in Westerlund 1, and therefore the result may not be a mass segregation that is limited to a particular mass range. Although the fluctuation is rather large, we view the trend in the radial variation of the IMFs as evidence of mass segregation. But since statistical uncertainties may dominate in the outer region due to the small number of massive stars, it is hard to measure the degree of mass segregation reliably. Violent processes among massive stars, such as dynamical ejection, mass loss from evolved stars, and SN explosions, may also affect the trend in the radial variation.

\section{DISCUSSION}

\subsection{The Presence of the Unexpectedly Faint Evolved Stars}

As mentioned in Section 3.4, the presence of relatively faint YHGs (W12a and W265) and RSGs (W20, W26, W75, and W237) is confusing. Owing to good seeing conditions, we could resolve W12a into two stars in the optical images. The star is about 1 mag fainter than the expected brightness from the stellar evolution model. According to Bonanos (2007), W12a, W265, W20, and W237 did not reveal photometric variability during seventeen nights. Neither could Clark et al. (2010) find any spectroscopic variability. However, Ritchie et al. (2009a) found photospheric pulsation in W265 and Clark et al. (2010) found variation in the spectral types of the RSGs. In our data, W20 was barely detected in the $V$ band, whereas the star was saturated in the $I$ band. For the case of W75, the star was only detected in the $I$ band. Given the limiting magnitude ( $V=24 \mathrm{mag}$ ) of our $V$-band data, W20 and W75 were much fainter than other RSGs (W26 and W237, $V \sim 17.5 \mathrm{mag}$ ). We also checked the brightness of the stars in the $K$ magnitude of Borgman et al. (1970). Obviously, W20 and W75 were about $\sim 1$ mag fainter than W26 or W237 in the $K$ band. 
As all previous studies noted, if the stars are physically connected to Westerlund 1, two issues need to be clarified. The first is whether or not differences in brightness are possibly due to variability. The Galactic YHG V509 Cas reveals photometric variations from $4.89 \mathrm{mag}$ to $5.18 \mathrm{mag}$ in the $V$ band (Percy \& Zsoldos 1992). The star gradually dimmed from 1980 to 1990 with, in addition, short-term fluctuations. An episodic variation of a Galactic YHG, $\rho$ Cas, in the $V$ band has also been reported by Lobel et al. (2003). The brightness suddenly dropped by $1.3 \pm 0.1 \mathrm{mag}$ in 2000. Therefore, the brightness of faint YHGs could be explained by variability. On the other hand, there are significant differences in the brightness among four RSGs as mentioned above. Since most RSGs are long-period variables (LPVs), the difference between faint (W20 and W75) and bright (W26 and W237) RSGs could be explained by pulsation if the stars are coeval. According to Kiss et al. (2006), the brightness of the extreme supergiant LPV VX Sgr varies by up to $5.8 \mathrm{mag}$ in the $V$ band. If W20 and W75 belong to this extreme class, the stars could be as bright as other RSGs in Westerlund 1 at maximum light. However, such an extreme class is very rare. Furthermore, the classification and the evolutionary status of VX Sgr is still uncertain as mentioned in Chiavassa et al. (2010). Wood et al. (1983) have studied the properties of a sample of LPV supergiants in the Small and Large Magellanic Clouds. They found a smaller mean pulsation amplitude of $\leqslant 0.25 \mathrm{mag}$ in $K$ for RSGs. Given the small variations of RSGs from the large sample, the difference of $\sim 1$ mag in $K$ among four RSGs in Westerlund 1 may not be explained by intrinsic variability, hence cluster membership of the two faint RSGs (W20 and W75) may be ruled out. To confirm this, photometric monitoring would be very useful.

The other issue is the presence of RSGs and the age of the cluster $(5.0 \pm 1.0 \mathrm{Myr})$. According to the stellar evolution models with rotation (Ekström et al. 2012), the RSGs with mass of $25 M_{\odot}$ are found at an age of $8 \mathrm{Myr}$. If they are cluster members, the age spread of Westerlund 1 is much larger than that discussed in Section 3.4. In the case of PMS stars in young open clusters, an age spread of $\Delta \tau_{\text {age,PMs }} \sim 4.0-6.0 \mathrm{Myr}$ is a typical value (see, e.g., Sung et al. 2000; Park et al. 2001; and Park \& Sung 2002). There are several uncertainties, such as differential reddening, the presence of accretion disks, star formation history within a cluster (Sung \& Bessell 2010), and in particular the dependency on the stellar evolution models or PMS evolution models. Unfortunately, the age spread of massive stars is poorly known due to several factors, such as rarity, high frequency of multiplicity, and the effect of rotation on the stellar evolution, etc. Consequently, if the age spread could be as large as that of PMS stars, Westerlund 1 might be formed by a single starburst but with a large age spread. We also note that uncertainties in the stellar evolution models of massive stars cannot be excluded. On the other hand, Kudryavtseva et al. (2012) have found a smaller age spread $(\Delta \tau \leqslant 0.4 \mathrm{Myr})$ for Westerlund 1 , while Sung \& Bessell (2004), Pang et al. (2011), and Rochau et al. (2010) found clues to a large age spread in another starburst cluster NGC 3603. The result of Kudryavtseva et al. (2012) seems statistically inadequate to conclude that the age spread in Westerlund 1 is very small because they used photometric data for a very narrow field of view. If their argument is correct, the faint evolved stars should be excluded from membership, or some other astrophysical interpretations are required to explain the range of stellar ages.

One possible scenario is related to a rather complicated history in the earliest epochs of star formation in Westerlund 1 as evidenced by two important observations. Ritchie et al. (2010) attempted to constrain the initial mass of the progenitor of the magnetar (CXO J164710.2-455216) from photometric and spectroscopic studies of the eclipsing binary W13 and suggested that the progenitor mass was larger than $45 M_{\odot}$. In the second observation, Kothes \& Dougherty (2007) found a large bubble with a minimum dynamical age of $2.5 \mathrm{Myr}$ in Westerlund 1, and suggested that the bubble was a sign of very early massive star evolution in the cluster. However, adopting a higher inclination angle would give a larger physical size for the bubble and subsequently a larger dynamical age of up to 5.0 Myr. Given the large age spread, the presence of a magnetar, and the dynamical age of the expanding bubble, significant star formation events may have occurred twice in Westerlund 1. The difference in the age of the RSGs $(\tau \geqslant 8.0 \mathrm{Myr})$ and Westerlund 1 ( $\tau=5.0 \pm 1.0 \mathrm{Myr}$ ) corresponds to the lifetime of the most massive star with a mass of $120 M_{\odot}$. The progenitors of RSGs and the relatively faint YHGs may have been formed together with the most massive stars in the giant molecular cloud. After 3.0-4.0 Myr, the most massive star would have exploded as an $\mathrm{SN}$, and the hot gas ejected from the $\mathrm{SN}$ explosion could be the bubble found by Kothes \& Dougherty (2007). The explosion may have induced the formation of second generation stars in the rest of the natal cloud. The current population of Westerlund 1 may have formed at this epoch. A similar star formation history had been suggested by Sung \& Bessell (2004) in another starburst cluster NGC 3603. The authors found an age difference between early-type stars in the core and halo, which implies two different star formation events. Indeed, Rochau et al. (2010) found the presence of PMS stars with a relatively old age from their proper motion study. The results by Kudryavtseva et al. (2012, see their Figure 2) and Pang et al. (2011) also suggested the presence of an older MS population both in the fields around the clusters Westerlund 1 and NGC 3603. To confirm such a star formation history in Westerlund 1 it will be necessary to study the low-mass stars in the halo of Westerlund 1 that are still in the PMS stage. Perhaps, confirmation of this scenario may give important information on the formation of starburst clusters. On the other hand, Gennaro et al. (2011) argued that the apparent elongation and mass segregation of the cluster could be explained by merging of multiple coeval subclusters in the same natal giant molecular cloud in the early phase of formation, given no evidence for a large age spread.

If the relatively low luminosities of the several evolved stars, W12a, W20, W26, W237, and W265, are not related to variability, we attribute the dimming to differential reddening. According to Lockwood (1974), the total extinction of W20 $\left(A_{V}=16.5 \mathrm{mag}\right)$ and W75 $\left(A_{V}=19.2 \mathrm{mag}\right)$ is higher than those of W26 $\left(A_{V}=12.1 \mathrm{mag}\right)$ and W237 $\left(A_{V}=13.6 \mathrm{mag}\right)$. Internal reddening by the extended material itself could cause such a difference in brightness among the RSGs. On the other hand, in the 8.6 GHz images of Dougherty et al. (2010), W20, $\mathrm{W} 26, \mathrm{~W} 12 \mathrm{a}$, and W265 reveal significant cometary shapes pointing toward the anti-center of the cluster, while W75 and W237 show no such asymmetrical structure. According to current stellar evolution models, the existence of such stars cannot be explained by a single burst of star formation $5 \mathrm{Myr}$ ago. Indeed, only a few RSGs can be statistically predicted from our simple simulations - a cluster with an age spread of $3 \mathrm{Myr}$ and the IMF similar to that of NGC 3603 or an age spread of 5 Myr and the Kroupa IMF. However, with such limited data we could not find strong evidence for the membership of these 
stars. Consequently, establishing or refuting, the membership of such stars will provide important clues to understanding the formation history of massive young clusters.

\subsection{The Shape of the Initial Mass Function}

The slope of the IMF in Figure 11 seems to be divided into three parts; $\log m \leqslant 1.0,1.0<\log m \leqslant 1.4$, and $\log m>1.4$. Given the result of Gennaro et al. (2011), the slope in the mass range $\log m<1.4$ may be similar to the Salpeter/Kroupa IMF or a slightly steeper IMF. For $1.0<\log m \leqslant 1.4$, the IMF shows a plateau, while the slope of the IMF for the most massive stars $(\log m>1.4)$ is very steep, as seen in other starburst clusters, e.g., the Arches cluster (Kim et al. 2007; Figer et al. 1999) and NGC 3603 (Sung \& Bessell 2004). However, the existence of a plateau is not certain and could have other explanations.

The IMF for low masses $(1.0 \lesssim \log m \lesssim 1.2)$ may be lower than the present value in Figure 11 if the IMF is compared to that of NGC 3603 or that derived from NIR photometry because it is strongly affected by the subtraction of the field star contribution. In general, the distribution of interstellar material within the Galactic disk is complex. Moreover, Westerlund 1 resides in the Scutum-Centaurus arm beyond the Sagittarius arm, and thus a non-uniform surface density of field stars is to be expected due to the superposition of two different spiral arms in the line of sight. Although the assumption that field stars are uniformly distributed in a given field of view is invariably assumed in order to select cluster members without independent membership criteria, a number of photometric studies of open clusters actually suffer from this statistical problem, to a greater or lesser degree. If the surface density of stars outside of the cluster region was underestimated, the resulting IMF would be steeper than the true IMF. In addition, reddening corrections and increasing photometric errors for faint stars could also contribute to the uncertainty in mass due to high levels of extinction, and the result may influence the shape of the IMF. On the other hand, the IMF obtained from NIR photometry for stars with masses below $10 M_{\odot}$ may be much more reliable than that from the optical photometry. Given that the shape of the IMF $(\log m \sim 1)$ is similar to that of NGC 3603, if the presence of the plateau in the IMF is real, other interpretations could be the presence of changes of slope in the IMF and/or a dependence on the calibration schemes.

There is practically no discussion in the literature on the presence of changes of slope in the IMF in high-mass regimes because in most young open clusters the number of massive stars $\left(m \geqslant 10 M_{\odot}\right)$ is very small. However, the number of massive stars in starburst clusters is not small, and so the result from such clusters is statistically meaningful.

Dib et al. (2007) attempted to reproduce the IMF of the Arches cluster from semi-analytical modeling. According to their work, the shape of the stellar IMF could be a result of the coalescence of pre-stellar cores (PSCs) at an early stage of cluster formation. The number of intermediate-mass PSCs rapidly decreases as they contract or merge with more massive PSCs. As a result, the stellar IMF of the Arches cluster reveals a plateau in the mass range $3-10 M_{\odot}$. In the case of Westerlund 1 , despite its similar shape IMF, the plateau appears in the mass range $10-25 M_{\odot}$. If the plateau in the IMF of Westerlund 1 is related to the depletion of intermediate-mass PSCs during the cluster formation stage as suggested by Dib et al. (2007), a different process may have operated in Westerlund 1. If we compare the age of three starburst clusters (the Arches cluster, NGC 3603, and Westerlund 1), the time evolution of the IMF can be excluded because the IMF of NGC 3603 is very similar to that of Westerlund 1, but the age of NGC 3603 is comparable to that of the Arches cluster. On the other hand, NGC 3603 and Westerlund 1 are located in a spiral arm, while the Arches cluster is at the Galactic center. If merging among PSCs effectively occurs near the Galactic center due to differences in the star formation environment, such as strong tidal forces, the plateau could have occurred at a relatively low mass range.

Several tables providing the relation between temperature and bolometric correction have been published. Unfortunately, a reliable calibration covering the complete spectral ranges is very rare. Due to the lack of many representative $\mathrm{O}$ stars and the use of inadequate LTE stellar atmospheric models for early O type stars, especially for supergiants, prior to 2000 , only limited information was available at the time. As the number of observed massive objects has increased and new atmospheric models taking into account non-LTE have emerged, a new and more reliable temperature scale and set of bolometric corrections for O3-09.5 stars have been provided by Martins et al. (2005). The temperature scale of Martins et al. (2005) is systematically lower than that of Flower (1977), Böhm-Vitense (1981), or Crowther (1997) for O type stars. Fortunately, since Flower's temperature scale is very similar to that of Martins et al. (2005) for late O type stars, a small discrepancy does not seriously affect our results. However, it is clear that discrepancies in effective temperature can be a factor resulting in systematic differences in bolometric correction, thereby producing different individual stellar masses. And therefore, the changes of the slope in the IMF of Westerlund 1 and NGC 3603 may be caused by the adopted temperature scale and bolometric correction calibration. In addition, in the calculation of the IMF of NGC 3603, Sung \& Bessell (2004) used the mass-luminosity relation from the stellar evolution models of Schaller et al. (1992); if the changes of the slope are not a real feature, they may also be caused by the mass-luminosity relation of massive stars used by the Geneva group.

\subsection{The Abnormal Color of W43c}

The $(B-V)$ color of the $\mathrm{O}$ type supergiant $\mathrm{W} 43 \mathrm{c}$ is very abnormal $(B-V \sim 2.0$; see Figure 4$)$, but its $(V-I)$ is fairly normal in the $(V, V-I)$ CMD. When we adopt the mean reddening of $\langle E(B-V)\rangle=4.19 \mathrm{mag}$, the intrinsic color of the star is $-2.18 \mathrm{mag}$. Given the intrinsic color $(B-V)_{0} \sim$ -0.33 mag of the hottest stars, a $B-V$ of -2.18 cannot be reached for a normal star. To identify the source of discrepancy, we have checked the optical images and photometric data.

The abnormal $B-V$ color is not caused by photometric error because the star is bright. Our $B-V$ of the star is consistent with that of Negueruela et al. (2010, see Table 1 of their paper). The residual $V$-band image after subtracting all the measured stars shows that W43c is an optical double star, while the $B$-band images show no sign of an optical double. Hence, we can attribute the cause of abnormal $(B-V)$ color to the superposition of a foreground star and a member (O9 Ib) of Westerlund 1 . To confirm this, we made a simple calculation by assuming that the purported stars have the same flux in the $V$ band because the two components in the residual image are very similar. The magnitude of W43c in the $V$ band was resolved into two using Pogson's formula. We also assume that the field star has $B-V=1.3 \mathrm{mag}$ and $V-I=2.0 \mathrm{mag}$, whereas the member of Westerlund 1 has $B-V=4.0 \mathrm{mag}$ and $V-I=5.0 \mathrm{mag}$. The deconvolution of the $V$ magnitude yields $B$ and $I$ magnitudes for each star, finally we calculated the composite magnitude in $B$ and 


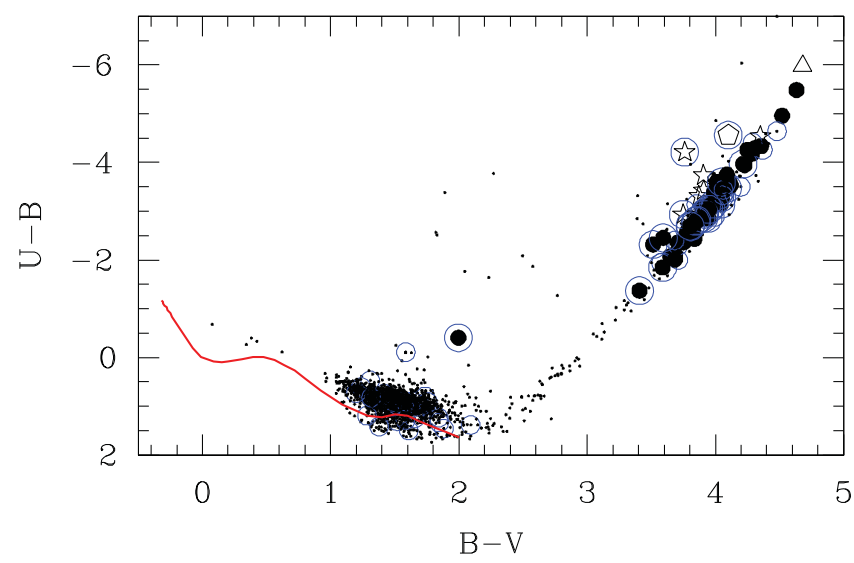

Figure 14. Effect of the red leak in the SDSS $u$ filter used with the MOSAIC II CCD attached to the Blanco $4 \mathrm{~m}$ telescope at CTIO. The solid line represents the intrinsic color relation for MS stars. There are two significant groups of stars in this diagram. The bluer group (in $B-V$ ) may be a less reddened foreground population, while the redder group (in $B-V$ ) consists of suspected members of Westerlund 1 . For those stars with $B-V>2.0$, the $U-B$ color indices become increasingly bluer due to the red leak of the SDSS $u$ filter.

(A color version of this figure is available in the online journal.)

$I$ of the system. As a result, the expected magnitudes are in good agreement with the observed ones, i.e., $\Delta B=-0.03$ mag and $\Delta I=-0.02 \mathrm{mag}$, where $\Delta$ is the expected magnitude minus the observed one. Consequently, the abnormal $B-V$ color of $\mathrm{W} 43 \mathrm{c}$ can be interpreted as the result of the superposition of a less-reddened foreground star and a highly reddened $\mathrm{O}$ type star in Westerlund 1.

\section{SUMMARY}

Starburst clusters are important objects to understand the properties, evolution, and formation of massive stars as well as the star formation in the early universe. Among several starburst clusters in the Galaxy, Westerlund 1 is the closest and the most massive cluster. We carried out $B V I_{C}$ and $J K_{S}$ photometry of Westerlund 1.

Using the spectral-type-intrinsic-color relation we obtained the color excess and total extinction in $V$, i.e., $\langle E(B-V)\rangle=$ $4.19 \pm 0.23 \mathrm{mag}$ and $\left\langle A_{V}\right\rangle=13.0 \pm 0.7 \mathrm{mag}$, respectively. The interstellar extinction in the NIR was also derived using the reddening law of Kim et al. (2006) and the EFL constructed in the $\left[M_{K_{S}},\left(J-K_{S}\right)_{0}\right]$ plane, i.e., $\left\langle E\left(J-K_{S}\right)\right\rangle=1.70 \pm 0.21 \mathrm{mag}$ and $A_{K_{S}}=1.12 \pm 0.14$ mag. Since the normal ZAMS fitting method was nearly impossible to apply to Westerlund 1 due to severe extinction, we fitted the EFL to the turn-on point from the PMS to the MS stars, thereby obtaining a distance of $3.8 \mathrm{kpc}$.

We constructed H-R diagrams using the conversion table of Flower (1977). By superimposing the new stellar evolution models with rotation of Ekström et al. (2012) on the H-R diagrams, an age of 5.0 $\pm 1.0 \mathrm{Myr}$ was obtained. The result is in good agreement with previous studies. However, the presence of RSGs could imply a large spread in the age of Westerlund 1. Hence, several plausible star formation histories were suggested.

The masses of evolved stars with spectral types were determined using the mass-luminosity relation provided by the stellar evolution models with rotation (Ekström et al. 2012). We also determined the mass of individual stars without spectral types from the relation between absolute magnitude and initial mass. Finally, the IMF of Westerlund 1 was obtained after statistically subtracting the contribution of field interlopers to the mass function. The slope of the IMF was found to be as shallow as that of
NGC 3603 center, when we used the complementary IMF derived from NIR photometry. We discussed possible changes of the slope in the IMF in different mass regimes in Westerlund 1.

The integration of the IMFs in the mass range $\left(85 M_{\odot}>\right.$ $m>0.08 M_{\odot}$ ) yielded a total masses of $7.3 \times 10^{4} M_{\odot}$ and $7.8 \times 10^{4} M_{\odot}$, respectively, where we assumed that the shape of the IMF in low-mass regime may be similar to that of NGC 2264 or that of Kroupa. Also, a lower limit of the total mass $\left(5.1 \times 10^{4} M_{\odot}\right)$ was derived from the radial variation of the IMF. If we assume this mass as the total mass, a half-mass radius is $1.1 \mathrm{pc}$ at $3.8 \mathrm{kpc}$. We confirm that Westerlund 1 is the most massive open cluster in the Galaxy.

The mass segregation of Westerlund 1 was also investigated and we found that the slope of the IMF varied systematically from the center to $r \sim 1$ '.7, but the pattern of variation changed at $r \simeq 1$ '.7. Some discussion was made concerning this issue.

The authors express deep thanks to the anonymous referee for many useful comments and suggestions. This work was partly supported by a National Research Foundation of Korean (NRF) grant funded by the Korea Government (MEST) (Grant No. 20120005318) and partly supported by the Korea Astronomy and Space Science Institute (KASI) (Grant No. 2011-9-300-02).

Facilities: Blanco, AAT

\section{APPENDIX A}

\section{THE RED LEAK IN THE SDSS $u$ FILTER}

Photometry in the $U$ band is of crucial important in reddening determination. Westerlund 1 is very reddened, but we had expected to detect the $U$ signal for a few of the brightest stars in Westerlund 1 with an exposure time of $1 \mathrm{hr}$ in the $u$ band. Contrary to our expectations, there were many bright stars suspected as members of Westerlund 1 in the images even in a 10 minute exposed image. Interestingly, extremely red stars revealed a double-peak profile in the surface plot. The difference in brightness between the two peaks was not as great as $0.1 \mathrm{mag}$, thus photometric data in the $u$ band were seen to be dominated by the red light leak, either resolved or not. Unfortunately, reliable extremely red standard stars for correcting this effect were absent. We confirmed the effect of the red leak on the $(U-B, B-V)$ diagram (Figure 14). The suspected members with the redder color strongly suffered from the red leak in the $U-B$ color. Bond (2005) mentioned that the red leak could be suppressed by adding a cutoff filter or by using a leak-free coated filter. In the work, the author also presented a procedure for correcting for the red leak. Unfortunately, due to the heavy reddening of Westerlund 1 , the resultant $U-B$ data would be very uncertain, even were the red leak corrected by the procedure.

\section{APPENDIX B}

\section{THE EMPIRICAL FIDUCIAL LINE}

The ZAMS relation is the most powerful tool in determining the distance to open clusters. Since the relation is based on the intrinsic properties of MS stars, that gives very accurate distance if the reddening toward a cluster is well determined. Unfortunately, the heavy extinction of Westerlund 1 does not permit us to determine the accurate distance using the ZAMS, or theoretical isochrones, because our detection limit $(V \sim$ $24 \mathrm{mag}$ ) is not faint enough to reach the gradual turning of the ZAMS, equivalent to A-F type stars. For this reason, we had to perform NIR photometry. 


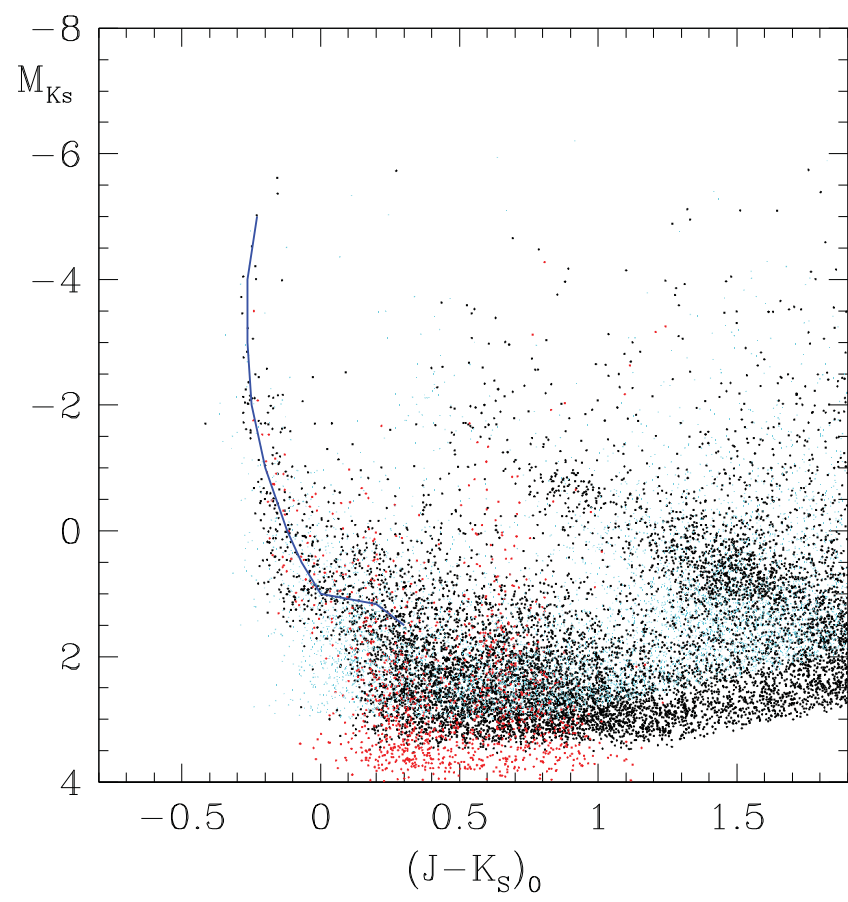

Figure 15. $M_{K_{S}}$ vs. $\left(J-K_{S}\right)_{0}$ color-magnitude diagram of three open clusters. Red, black, and cyan represent the stars in NGC 2362, NGC 6231, and NGC 6823, respectively. We set an empirical fiducial line (solid line) based on the photometric data. See Appendix B for details.

What will be used here is similar to the isochrone placement as shown in Brandner et al. (2008) and Gennaro et al. (2011), but a more empirical approach as for the ZAMS relation. First, we suppose the age of Westerlund 1 to be 3.0-6.0 Myr from previous studies. Open clusters with a similar age were searched for. NGC 2362 (3.0-6.0 Myr; Moitinho et al. 2001), NGC 6231 (2.5-5.0 Myr; Santos \& Bica 1993; Sung et al. 1998; Baume et al. 1999), and NGC 6823 (2.0-7.0 Myr; Bica et al. 2008) were selected for constructing the empirical relation. Subsequently, $U B V$ CCD photometric data of each of the clusters were obtained from WEBDA (http://www.univie.ac.at/webda/), and we adopted the distance and reddening from Delgado et al. (2006) for NGC 2362, Sung et al. (1998) for NGC 6231, and Guetter (1992) for NGC 6823. NIR data for these clusters were obtained from the 2MASS catalog (Skrutskie et al. 2006). To place NIR photometric data of stars belonging to the cluster in the $\left[M_{K_{S}},\left(J-K_{S}\right)_{0}\right]$ plane, we needed to determine the reddening in the NIR region. Using the intrinsic color relations, i.e., $(B-V)_{0}$ versus $(V-\lambda)_{0}, E(V-J)$, and $E\left(V-K_{S}\right)$ were determined, thereby $E\left(J-K_{S}\right)$ and $A_{K_{S}}$ were obtained by adopting the $A_{V}$ values from the literature. We present the results in Figure 15. In the case of NGC 2362, the reddening is so small that the total extinction $A_{K_{S}}$ would be strongly affected by statistical fluctuations, so we assumed $A_{K_{S}}=0.0$ for NGC 2362. As all NIR data of the clusters were corrected for reddening and distance, the CMDs could be superimposed in the photometric plane on an absolute scale. Finally, we drew the best-fitting line, which traced the middle of the MS and PMS sequences, and present it in Figure 15. We call this the empirical fiducial line.

\section{REFERENCES}

Allison, R. J., Goodwin, S. P., Parker, R. J., Portegies Zwart, S. F., \& de Grijs, R. 2010, MNRAS, 407, 1098
Allison, R. J., Goodwin, S. P., Parker, R. J., et al. 2009, ApJL, 700, 99 Baume, G., Vázquez, R. A., \& Feinstein, A. 1999, A\&AS, 137, 233 Bica, E., Bonatto, C., \& Dutra, C. M. 2008, A\&A, 489, 1129 Böhm-Vitense, E. 1981, ARA\&A, 19, 295

Bonanos, A. Z. 2007, AJ, 133, 2696

Bond, H. E. 2005, AJ, 129, 2914

Bonnell, I. A., \& Bate, M. R. 2006, MNRAS, 370, 488

Bonnell, I. A., Bate, M. R., \& Zinnercker, H. 1998, MNRAS, 298, 93

Bonnell, I. A., \& Davies, M. B. 1998, MNRAS, 295, 691

Borgman, J., Koornneef, J., \& Slingerland, J. 1970, A\&A, 4, 248

Brandner, W., Clark, J. S., Stolte, A., et al. 2008, A\&A, 478, 137

Chiavassa, A., Lacour, S., Millour, F., et al. 2010, A\&A, 511, 51

Clark, J., Richie, B. W., \& Negueruela, I. 2010, A\&A, 514, A87

Clark, J., Richie, B. W., Negueruela, I., et al. 2011, A\&A, 531, A28

Clark, J. S., Fender, R. P., Waters, L. B. F. M., et al. 1998, MNRAS, 299, L43

Clark, J. S., Muno, M. P., Negueruela, I., et al. 2008, A\&A, 477, 147

Clark, J. S., \& Negueruela, I. 2002, A\&A, 396, L25

Clark, J. S., \& Negueruela, I. 2004, A\&A, 413, L15

Clark, J. S., Negueruela, I., Crowther, P. A., \& Goodwin., S. P. 2005, A\&A, 434 949

Crowther, P. A. 1997, in IAU Symp. 189, Fundamental Stellar Properties: The Interaction between Observation and Theory, ed. T. R. Bedding, A. J. Booth, \& J. Davis (Dordrecht: Kluwer), 137

Crowther, P. A., Hadfield, L. J., Clark, J. S., Negueruela, I., \& Vacca, B. D. 2006, MNRAS, 372, 1407

Delgado, A. J., González-Martín, O., Alfaro, E. J., \& Yun, J. 2006, ApJ, 646, 269

Dib, S., Kim, J., \& Shadmehri, M. 2007, MNRAS, 381, L40

Dib, S., Shadmehri, M., Gopinathan, M., Kim, J., \& Henning, Th. 2008, in ASP Conf. Ser. 387, Massive Star Formation: Observations Confront Theory, ed. H. Beuther, H. Linz, \& T. Henning (San Francisco, CA: ASP), 282

Dougherty, S. M., Clark, J. S., Negueruela, I., Johnson, T., \& Chapman, J. M. 2010, A\&A, 511, A58

Eisenhauer, F., Quirrenbach, A., Zinnecker, H., \& Genzel, R. 1998, ApJ, 498, 278

Ekström, S., Georgy, C., Eggenberger, P., et al. 2012, A\&A, 537, 146

Espinoza, p., Selman, F. J., \& Melnick, J. 2009, A\&A, 501, 563

Figer, D. F., Kim, S., Morris, M., et al. 1999, ApJ, 525, 750

Flower, P. J. 1977, A\&A, 54, 31

Gennaro, M., Brandner, W., Stolte, A., \& Henning, Th. 2011, MNRAS, 412,2469

Guetter, H. H. 1992, AJ, 103, 197

Hillenbrand, L. A., \& Hartmann, L. W. 1998, ApJ, 492, 540

Hosokawa, T., Offner, S. S. R., \& Krumholz, M. R. 2011, ApJ, 738, 140

Humphreys, R. M., \& Davidson, K. 1979, ApJ, 232, 409

Hur, H., Sung, H., \& Bessell, M. S. 2012, AJ, 143, 41

Kim, S., Figer, D. F., Kudritzki, R. P., \& Najarro, F. 2007, JKAS, 40, 153

Kim, S., Figer, D. F., \& Lee, M. G. 2006, PASP, 118, 62

Kiss, L. L., Szabó, G. M., \& Bedding, T. R. 2006, MNRAS, 372, 1721

Kook, S.-H., Sung, H., \& Bessell, M. S. 2010, JKAS, 43, 141

Koornneef, J. 1977, A\&A, 55, 469

Kothes, R., \& Dougherty, S. M. 2007, A\&A, 468, 993

Koumpia, E., \& Bonanos, A. Z. 2012, A\&A, 547, 30

Kroupa, P. 2002, Sci, 295, 82

Kudryavtseva, N., Brandner, W., Gennaro, M., et al. 2012, ApJL, 750, 44

Landolt, A. U. 1992, AJ, 104, 340

Lim, B., Sung, H., Bessell, M. S., Karimov, R., \& Ibrahimov, M. 2009, JKAS, $42,161 \mathrm{~L}$

Lobel, A., Dupree, A. K., Torres, G., et al. 2003, ApJ, 583, 923

Lockwood, G. W. 1974, ApJ, 193, 103

Magnier, E. A., \& Cuillandre, J.-C. 2004, PASP, 116, 449

Martins, F., Schaerer, D., \& Hillier, D. J. 2005, A\&A, 436, 1049

Massey, P. 2003, arXiv:astro-ph/0307531v1

Massey, P., \& Hunter, D. A. 1998, ApJ, 493, 180

Mayne, N. J., Naylor, T., Littlefair, S. P., Saunders, E. S., \& Jeffries, R. D. 2007, MNRAS, 375, 1220

McMillan, S. L. W., Vesperini, E., \& Portegies Zwart, S. F. 2007, ApJL, 655, 45 Mengel, S., \& Tacconi-Garman, L. E. 2007, A\&A, 466, 151

Moeckel, N., \& Bonnell, I. A. 2009, MNRAS, 400, 657

Moitinho, A., Alves, J., Huélamo, N., \& Lada, C. J. 2001, ApJL, 563, 73

Muno, M. P., Law, C., Clark, J. S., et al. 2006, ApJ, 650, 203

Negueruela, I., Clark, J., \& Richie, B. W. 2010, A\&A, 516, 78

Pang, X., Pasquali, A., \& Grebel, E. K. 2011, AJ, 142, 132

Park, B.-G., \& Sung, H. 2002, AJ, 123, 892

Park, B.-G., Sung, H., \& Kang, Y. H. 2001, JKAS, 34, 149

Percy, J. R., \& Zsoldos, E. 1992, A\&A, 263, 123

Piatti, A. E., Bica, E., \& Claria, J. J. 1998, A\&AS, 127, 423 
Price, S. D. 1968, AJ, 73, 431

Raboud, D., \& Mermilliod, J.-C. 1998, A\&A, 333, 897

Rieke, G. H., \& Lebofsky, M. J. 1985, ApJ, 288, 618

Ritchie, B. W., Clark, J. S., Negueruela, I., \& Crowther, P. A. 2009a, A\&A, 507,1585

Ritchie, B. W., Clark, J. S., Negueruela, I., \& Langer, N. 2010, A\&A, 520, A48

Ritchie, B. W., Clark, J. S., Negueruela, I., \& Najarro, F. 2009b, A\&A, 507, 1597

Rochau, B., Brandner, W., Stolte, A., et al. 2010, ApJL, 716, 90

Santos, J. F. C., \& Bica, E. 1993, MNRAS, 260, 915

Schaller, G., Schaerer, D., Meynet, G., \& Maeder, A. 1992, A\&AS, 96, 269

Skinner, S. L., Simmons, A. E., Zhekov, S. A., et al. 2006, ApJL, 639, 35

Skrutskie, M. F., Cutri, R. M., Stiening, R., et al. 2006, AJ, 131, 1163

Stetson, P. 2000, PASP, 112, 773

Stolte, A., Brandner, W., Brandl, B., \& Zinnecker, H. 2006, AJ, 132, 253
Sung, H., \& Bessell, M. S. 1999, MNRAS, 306, 361

Sung, H., \& Bessell, M. S. 2000, PASA, 17, 244

Sung, H., \& Bessell, M. S. 2004, AJ, 127, 1014

Sung, H., \& Bessell, M. S. 2010, AJ, 140, 2070

Sung, H., Bessell, M. S., Chun, M.-Y., Karimov, R., \& Ibrahimov, M. 2008, AJ, 135,441

Sung, H., Bessell, M. S., Lee, H.-W., Kang, Y. H., \& Lee, S.-W. 1999, MNRAS, 310,982

Sung, H., Bessell, M. S., \& Lee, S.-W. 1997, AJ, 114, 2644

Sung, H., Bessell, M. S., \& Lee, S.-W. 1998, AJ, 115, 734

Sung, H., Chun, M.-Y., \& Bessell, M. S. 2000, AJ, 120, 333

Sung, H., Sana, H., \& Bessell, M. S. 2013, AJ, in press (arXiv:1211.4278)

Westerlund, B. 1961, PASP, 73, 61

Westerlund, B. 1968, ApJ, 154, 67

Westerlund, B. 1987, A\&AS, 70, 311

Wood, P. R., Bessell, M. S., \& Fox, M. W. 1983, ApJ, 272, 99 